\title{
EXPERIMENTAL MEASUREMENTS OF THE FUELLING EFFICIENCY OF IMPURITIES INJECTED INTO TEXTOR
}

\author{
G.M. McCRACKEN $+*$, U. SAMM, P.C. STANGEBY ${ }^{++}$, G. BERTSCHINGER, \\ J.A. BOEDO, S.J. DAVIES, D.S. GRAY, V. PHILIPPS, R.A. PITTS**, \\ D.H.J. GOODALL ${ }^{+}$, A. POSPIESZCZYK, R.P. SCHORN, B. SCHWEER, \\ G. TELESCA, B. UNTERBERG, G. WAIDMANN \\ Institut für Plasmaphysik, \\ Forschungszentrum Jülich $\mathrm{GmbH}$, \\ Association Euratom/KFA, \\ Jülich, Germany \\ + AEA Fusion (Euratom/UKAEA Fusion Association), \\ Culham Laboratory, \\ Abingdon, Oxfordshire, \\ United Kingdom \\ ++ University of Toronto, \\ Institute for Aerospace Studies, \\ Downsview, Ontario, \\ Canada
}

\begin{abstract}
Impurity fuelling has been studied in TEXTOR by gas puffing under a wide range of experimental conditions. First, neon has been injected into discharges with a range of densities during both Ohmic and neutral injection heating. Then, helium, neon and argon have been injected into similar discharges and the increase in radiated power and $Z_{\text {eff }}$ studied as a function of the number of impurity atoms injected. Similar experiments using carbon monoxide and methane yielded markedly different results from those of the rare gases. The fraction of injected atoms entering the confined plasma has been estimated and found to vary widely, from less than $1 \%$ for carbon to about $100 \%$ for helium. Detailed Monte Carlo calculations performed using the LIM code to simulate the impurity behaviour have shown that good agreement with experiment can be obtained for all species except oxygen, where charge exchange processes are thought to be important.
\end{abstract}

\section{INTRODUCTION}

The impurity content of a tokamak depends not only on the rate at which impurities are introduced but also on the probability that they actually enter the confined plasma, where they can contribute to fuel dilution and radiation. Although considerable attention has been given to impurity transport in the centre of the plasma $[1,2]$ and to local transport in low ionization states in the plasma boundary [3-5], little effort has been directed towards the direct measurement of the relationship between the number of impurity atoms injected and the central impurity concentration.

In the present paper we describe a number of detailed experiments in which the absolute fuelling efficiency has

\footnotetext{
Present affiliations:

* Plasma Fusion Center, Massachusetts Institute of Technology, Cambridge, MA, United States of America.

** Centre de Recherches en Physique des Plasmas, Lausanne, Switzerland.
}

been measured for a range of different species as a function of fuelling rate and of plasma parameters. Estimates have been made of the central impurity concentration from the measurements of $Z_{\text {eff }}$ and of the edge concentrations using both the total radiation and the spectroscopy of various charge states. The time constants of the various parameters have been studied and used to derive estimates of the impurity replacement times and recycling coefficients. Large differences in behaviour have been observed for different impurity species and with different plasma conditions.

\section{MODELLING}

There is no widely accepted definition of the concept of fuelling efficiency. Owing to recycling processes in the plasma boundary and the possibility of atoms being trapped in the limiters or wall surfaces, an atom may either have many chances of reaching the last closed 
flux surface (LCFS) or it may be lost after one or more recycling times.

In order to establish a framework in which to interpret the experimental data, a simple global model is considered. Let the primary flux of injected species be $G$ particles $\cdot \mathrm{s}^{-1}$. A fraction of these is trapped in the scrape-off layer (SOL) so that a flux $S$ reaches the confined plasma. We assume that the impurities recycle at the limiter surface with a probability $R$ and that they have a replacement (or global recycling) time, $\tau_{\mathrm{p}}$. It is assumed that for practical purposes, because of the grazing incidence of ions striking the limiter, all particles which recycle at the limiter re-enter the confined plasma. This is not strictly true as some small fraction may escape to the wall. A more detailed model has been described by Weynants et al. [6]. We consider the familiar global recycling equation, first used to describe the main plasma species [7]. The total impurity content $N$ is given by

$\frac{\mathrm{d} N}{\mathrm{~d} t}=-\frac{N}{\tau_{\mathrm{p}}}+\frac{R N}{\tau_{\mathrm{p}}}+S=-\frac{N}{\tau_{\mathrm{p}}^{*}}+S$

where

$\tau_{\mathrm{p}}^{*}=\tau_{\mathrm{p}} /(1-R)$

Note that all the symbols here refer to the impurities. Recycling of impurities from the wall will be negligible because their charge exchange cross-section is low.

Provided $R$ is close to unity $\tau_{\mathrm{p}}^{*}$ will be large compared with the time constants arising from transport within the plasma. In our results we find that for all cases, except carbon, $R$ is sufficiently high for this assumption to be justified. Experimental evidence on diffusion rates comes from experiments on helium transport both in TEXTOR [8] and in TFTR [9], where the effective time constants for radial transport are typically less than $0.1 \mathrm{~s}$. Provided diffusion is predominantly anomalous rather than classical it is expected that diffusion rates will be independent of impurity mass and charge [10].

When $S$ is constant the solution to Eq. (1) is

$N(t)=S \tau_{\mathrm{p}}^{*}+\left(N_{0}-S \tau_{\mathrm{p}}^{*}\right) \exp \left(-t / \tau_{\mathrm{p}}^{*}\right)$

where $N_{0}$ is the initial impurity content. For $N_{0}=0$ and $t \ll \tau_{\mathrm{p}}^{*}$ we see that $N$ should increase linearly with time, giving a direct measure of the flux source $S$. For $t \gg \tau_{\mathrm{p}}^{*}, N=S \tau_{\mathrm{p}}^{*}$. A value of $\tau_{\mathrm{p}}^{*}$ can be obtained directly from the time dependence of $N$, either when the source is present and the concentration is building up to the equilibrium value, or when the source function is removed and the concentration is decaying.
It is possible that this simple model will not give a reasonable representation of the impurity behaviour either because the recycling coefficient may change with time or because the injection of impurities may so alter the boundary conditions that $\tau_{\mathrm{p}}$ changes. In practice, it has been found to describe the behaviour of the rare gases reasonably well. It is, however, already clear that the number of particles in the confined plasma depends on time, the recycling coefficient and $\tau_{\mathrm{p}}$. Through $\tau_{\mathrm{p}}$ there will be a dependence on crossfield diffusion, $D_{\perp}$, and on ionization mean free path, $\lambda_{\mathrm{i}}$ [5] (cf. Section 5.2).

Further information can be obtained from the absolute value of the recycling flux, which can be measured experimentally. Let us define the recycling flux as

$\Gamma_{R}(t)=\frac{R N(t)}{\tau_{\mathrm{p}}}$

If in steady state conditions the total influx, $\Gamma_{R}(t)+S$, can be measured, then from Eq. (1) we can obtain a direct measure of $\tau_{\mathrm{p}}$. The total impurity concentration is obtained from $Z_{\text {eff }}$ as discussed in Section 4.1. From the definition of $\tau_{\mathrm{p}}^{*}$, Eq. (2), $R$ can be calculated. When conditions are not steady state, provided $S$ is constant, the solution for $N(t)$ from Eq. (3) may be substituted into Eq. (4). Taking the case where $N_{0}=0$ we obtain

$\frac{\Gamma_{R}(t)}{S}=\frac{R}{1-R}\left[1-\exp \left(-t / \tau_{\mathrm{p}}^{*}\right)\right]$

Thus, the ratio of the recycling flux to the primary flux $S$ provides a measure of $R$. An integral form of Eq. (5) can also be derived if $S$ is not constant.

We still have to discuss the relationship between the influx of gas $G$ and the effective source function $S$. The ratio of $S / G$ will clearly depend on the extent of ionization in the SOL and surface trapping in the walls and limiter surfaces. If an incoming atom is ionized in the SOL then it may diffuse across the field to enter the plasma. The probability of this is low but is given explicitly by the Engelhardt model [5, 11]. Alternatively, the ion may travel along field lines until it impinges on the limiter. Owing to its incident energy (sheath plus thermal), there is a possibility that the ion may be trapped in the surface or that it may be released as a neutral with another chance of reaching the LCFS. The recycling coefficient from this surface, $R_{s}$, is not necessarily the same as the recycling coefficient at the limiter front surface. Because the particle flux decreases with radius, the limiter surface closer to the wall and the wall itself are less likely to be saturated by implanted impurities and so, in general, it would be expected that 
$R_{\mathrm{S}}<R$. The ratio of $S / G$ will thus be a complicated function depending on the parameters $D_{\perp}, \lambda_{\mathrm{i}}, R_{\mathrm{s}}$, etc., none of which are readily accessible for modelling purposes. However, the ratio $S / G$ can be determined experimentally.

In earlier experiments [12] it was found that $S / G$ varied widely with different gases. It may also vary with time because of changes in surface trapping. One of the main objectives of the present study is to show how the effective source function $S$ changes with impurity gas species and plasma conditions.

\section{EXPERIMENT AND DIAGNOSTICS}

Operations at TEXTOR [13] have used steady state plasma conditions, which in typical experiments were: $\bar{n}_{\mathrm{e}}=(1.5-4.5) \times 10^{19} \mathrm{~m}^{-3}, I_{\mathrm{p}}=350 \mathrm{kA}$ and $T_{\mathrm{e}}(0)=1 \mathrm{keV}$, with deuterium as the main plasma species. Some experiments with neutral beam heating have also been carried out. The impurity gas is injected both from the wall position, using an accurately calibrated gas flow, and through a hole in a test limiter whose minor radius can be varied from the wall position $(r=0.52 \mathrm{~m})$ to a position inside the main ALT II pumped limiter [14] at $r=0.46 \mathrm{~m}$. Operations with ALT II normally have the pumps open, providing net pumping for both plasma species and impurities. However, these pumps can be shut and the intrinsic pumping of the impurities investigated. A schematic diagram of the main diagnostics of interest and of the gas puffing positions is shown in Figs 1(a) and (b).

The main plasma gas feed is controlled by a feedback loop to keep the line average density constant. From the reduction of the flow through this feed valve it is possible to measure the flux of electrons into the plasma due to the impurity gas flow. This provides an independent measure of the fuelling efficiency of the impurity flux relative to that of deuterium.

The flow from the gas valve at the wall position is calibrated to an accuracy of better than $5 \%$ and the time dependence can be preprogrammed. Gas is normally injected at a constant flow rate for a fixed period of typically 0.5 to $1.0 \mathrm{~s}$ during the steady state period of the discharge, but it has also been operated in feedback mode using a spectroscopic signal to maintain a steady state impurity concentration [15]. The density and temperature are typically constant from $0.5-2.0 \mathrm{~s}$. The gas flow through the limiter is not so well controlled, having a flow rate that varies with time during the injection pulse and a relatively long decay after the gas valve is

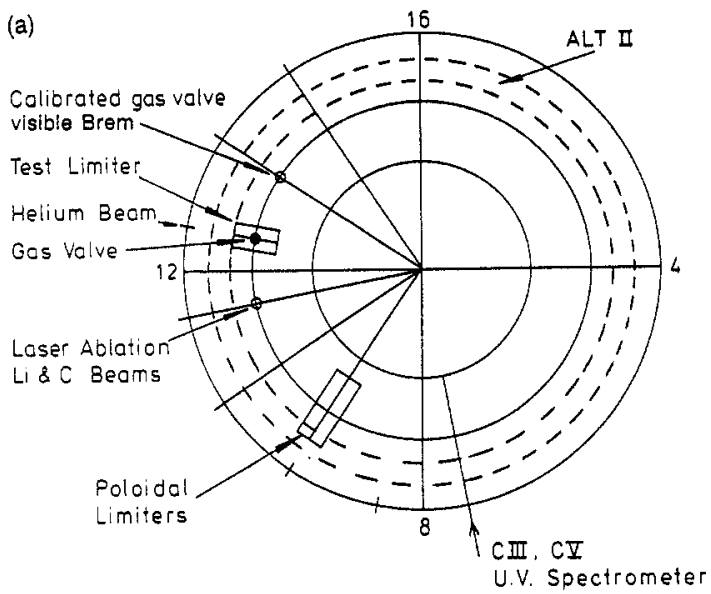

(b)

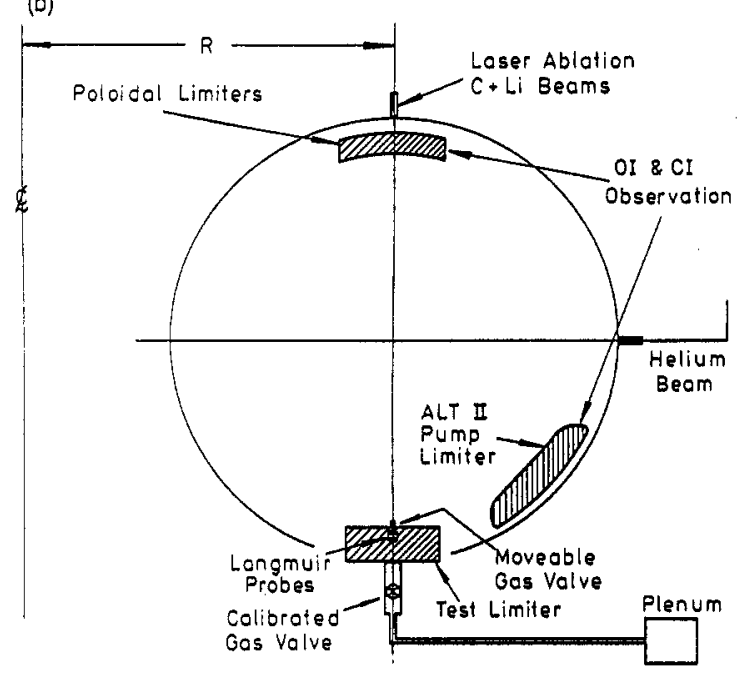

FIG. 1. Schematic diagram of experimental arrangement.

nominally closed. The integral of the flow over the injection period can, however, be reliably measured.

Impurity fluxes leaving the limiters are monitored using photomultipliers fitted with optical interference filters for O I, C I, $\mathrm{D}_{\alpha}$, He I and Ne I. Visible and UV spectrometers are also used routinely to measure the line integral of other suitable spectroscopic lines, in particular Ne III and Ne VIII. The radial profile of the total radiation is measured with an eight channel bolometer array and the central radial profiles $n_{\mathrm{e}}(r)$ and $T_{\mathrm{e}}(r)$ are measured with an interferometer array and an ECE system, respectively. Detailed $n_{e}$ and $T_{\mathrm{e}}$ edge profiles are measured using lithium, carbon and helium beams $[16,17]$. In some runs additional measurements of edge profiles were made using a reciprocating Langmuir probe [18]. The $Z_{\text {eff }}$ is calculated from the plasma conductivity using the measured value of $T_{\mathrm{e}}(0)$. In some cases independent measurements of $Z_{\text {eff }}$ have been made using visible bremsstrahlung [19]. 


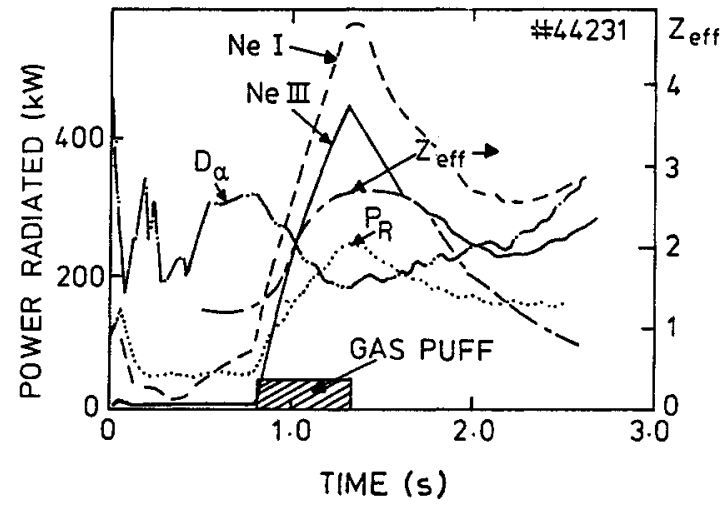

FIG. 2. Time dependences of $\mathrm{P}_{\mathrm{R}}, Z_{e f f}$, Ne I, Ne III and $D_{\alpha}$, during and after a $0.5 s$ neon gas puff into a $D_{2}$ discharge with constant density. The ALT II pumps were on, $\mathrm{I}_{p}=350 \mathrm{kA}$ and $\mathrm{n}_{e}=2 \times 10^{19} \mathrm{~m}^{-3}$.

\section{RESULTS}

\subsection{Neon}

A typical result for a $0.5 \mathrm{~s}$ neon injection at constant flow rate into a deuterium discharge is shown in Fig. 2. As shown by the Ne III line at $268.3 \mathrm{~nm}$ the neon recycling at the limiter increases approximately linearly during the gas injection. The radiated power, $Z_{\text {eff }}$ and $\mathrm{Ne} I$ all increase roughly proportionally to the Ne III. At the end of the gas injection the same signals decay exponentially until the time when the plasma current and density begin to ramp down $(\sim 2.0 \mathrm{~s})$. The hydrogen recycling signals at ALT II and the poloidal limiter decay during neon injection, indicating fuel dilution at the constant density imposed. The torus had been boronized [20] a few days before these experiments, and a typical value of $Z_{\text {eff }}$ before neon injection was 1.3.

Similar measurements obtained by injecting neon at the same flow rate into discharges with different densities are shown in Fig. 3. Results are presented for the $\mathrm{Ne}$ VIII emission in both ohmically heated and neutral beam heated discharges. Following impurity injection the decay is in each case a good approximation to an exponential until at least $2.5 \mathrm{~s}$ for the Ohmic case and until $2.0 \mathrm{~s}$ in the case of NBI. The time constants, $\tau_{\mathrm{p}}^{*}$, calculated from the $\mathrm{Ne} \mathrm{I}$ and $\mathrm{Ne}$ VIII signals are plotted in Fig. 4. They do not vary significantly with density but there is a marked difference between the Ohmic and NBI cases. This is discussed in Section 5.2. The results for Ne I and Ne VIII are similar and are consistent with earlier measurements of $\tau_{\mathrm{p}}^{*}$ for neon in
TEXTOR Ohmic discharges (see Ref. [6]). These measurements were made with the ALT II pumps on and so are in effect a measure of the efficiency of the pumped limiter. The data with the pumped limiter off are discussed in Section 4.3.

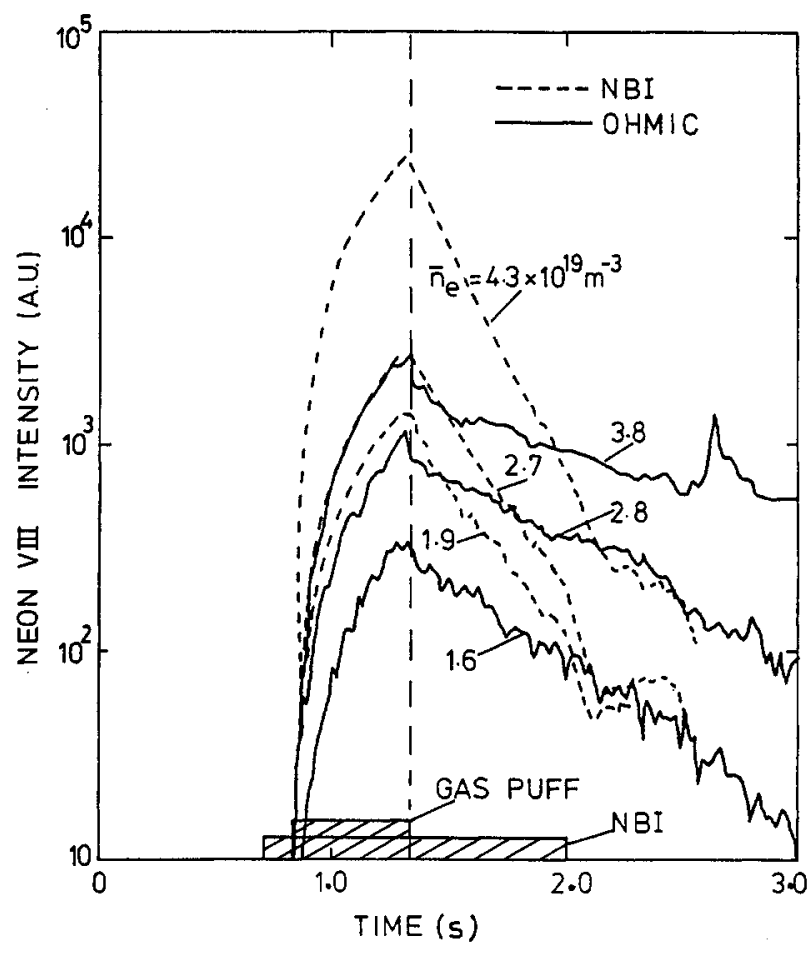

FIG. 3. Time dependence of the Ne VIII emission for different densities during and after a neon gas puff with Ohmic heating and with $1.5 \mathrm{MW} \mathrm{NBI}$. Deuterium discharge, $\mathrm{I}_{p}=350 \mathrm{kA}$ and gas flow rate was $2 \times 10^{18}$ atoms $\cdot \mathrm{s}^{-1}$ for Ohmic and $3.7 \times 10^{18}$ atoms $\cdot \mathrm{s}^{-1}$ for $N B I$.

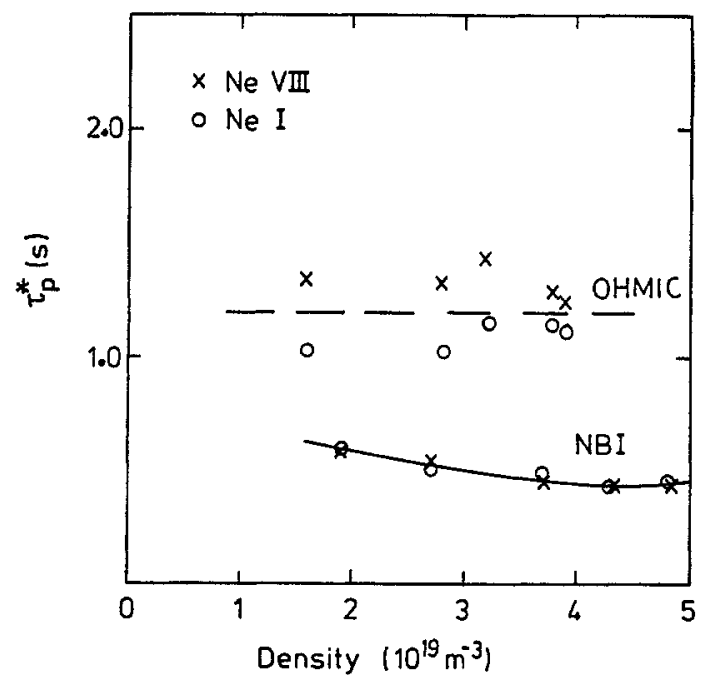

FIG. 4. Variation of $\tau_{p}^{*}$ with density in Ohmic and neutral beam heated discharges derived from the spectroscopic signals of $\mathrm{Ne} I$ and Ne VIII. 


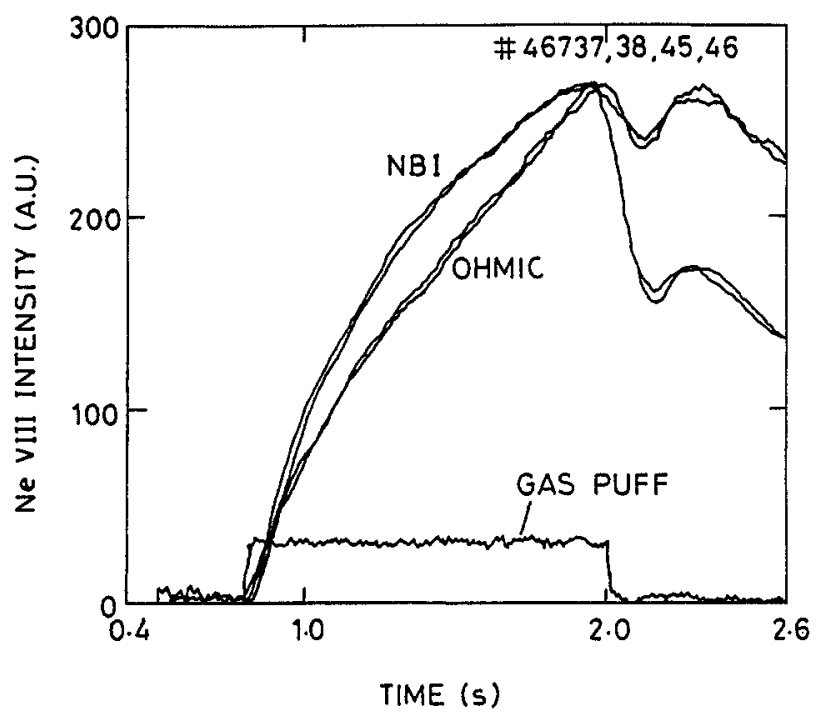

FIG. 5. Time dependence of the normalized Ne VIII emission during a long gas puff into deuterium discharges with Ohmic and NBI heating. The time of the gas puffing, 0.8 to $2.0 \mathrm{~s}$, is indicated. The electron density was $\mathrm{n}_{e}=2.5 \times 10^{19} \mathrm{~m}^{-3}$ for Ohmic and $2.7 \times 10^{I 9} \mathrm{~m}^{-3}$ for $\mathrm{NBI}$.

Time constants have been derived from the rising part of the curves during gas injection, $t=0.8-1.3 \mathrm{~s}$ in Fig. 3, using Eq. (3). Because $t<\tau_{\mathrm{p}}^{*}$ the curve is not very sensitive to changes in $\tau_{\mathrm{p}}^{*}$. Within the uncertainties, no difference can be detected between results at different densities, but the values obtained are consistent with those obtained from the decay after $1.3 \mathrm{~s}$. In a few cases the gas injection was continued to $2.0 \mathrm{~s}$, allowing the system to approach closer to equilibrium. The results are shown in Fig. 5. The difference in $\tau_{\mathrm{p}}^{*}$ between the Ohmic and NBI cases in the rising part of the curve is now evident. The two Ohmic curves have injection rates differing by a factor of 2 and there is no detectable difference between the values of $\tau_{\mathrm{p}}^{*}$ in the two cases.

The discharge time was too short to reach steady state conditions by natural evolution, but by applying feedback from the $\mathrm{Ne}$ VIII signal to the gas inlet system, steady state conditions could be rapidly produced [15]. This is illustrated for $\mathrm{Ne} \mathrm{I}$ and $\mathrm{Ne}$ VIII for an NBI discharge in Fig. 6. A similar result is obtained in Ohmic discharges. As far as can be determined, all signals have reached steady state. The Ne I signal is measured at the limiter and is absolutely calibrated in atomic flux $\cdot \mathrm{m}^{-1} \cdot \mathrm{s}^{-1}$ at $T_{\mathrm{e}}=30 \mathrm{eV}$. The photon efficiency for the neutral lines is calculated using the technique previously described [21]. The edge temperature stays roughly constant in these experiments and so the effect of changing edge temperature is not included. By assuming toroidal symmetry along the length of the ALT II limiter we can obtain a measure of the total neon recycling flux. Since conditions are steady we can use Eq. (1) to obtain $\tau_{\mathrm{p}}$, provided that we can calculate the total neon inventory in the plasma. This is obtained from $Z_{\text {eff }}$ assuming that the $Z_{\text {eff }}$ profile is flat and that the neon is fully ionized, a good assumption for $T_{\mathrm{e}}>0.5 \mathrm{keV}$. The conductivity $Z_{\text {eff }}$ is used and Spitzer resistivity is assumed. The calculated value of $\tau_{\mathrm{p}}$ is $100 \mathrm{~ms}$ for high density Ohmic discharges and $8 \mathrm{~ms}$ for neutral beam heated discharges. There is a possible error of at least a factor of 2 in these results, due to the uncertainty in the absolute recycling flux and to the assumed uniformity of $Z_{\text {eff. }}$.

\subsection{Effect of impurity source position}

The simple Engelhardt 1-D model of transport [11] predicts that the central concentration of impurities should depend on the point of ionization, which in turn ought to depend on the position of the gas source. In order to investigate this effect, a number of experiments have been carried out using gas fuelling through a hole in a test limiter, which can be moved in radial position.

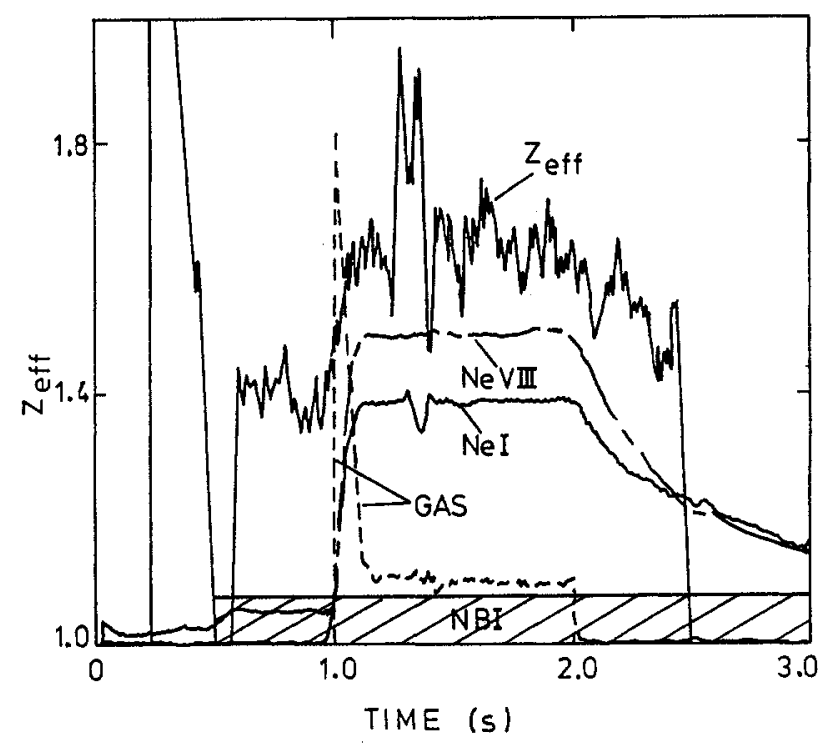

FIG. 6. Ne I, Ne VIII, $\mathrm{Z}_{\text {eff }}$ and neon flow rate during neon gas puffing into a deuterium discharge with feedback control of the Ne VIII: $\mathrm{n}_{e}=2.7 \times 10^{19} \mathrm{~m}^{-3}$, neutral beam heated discharge. 


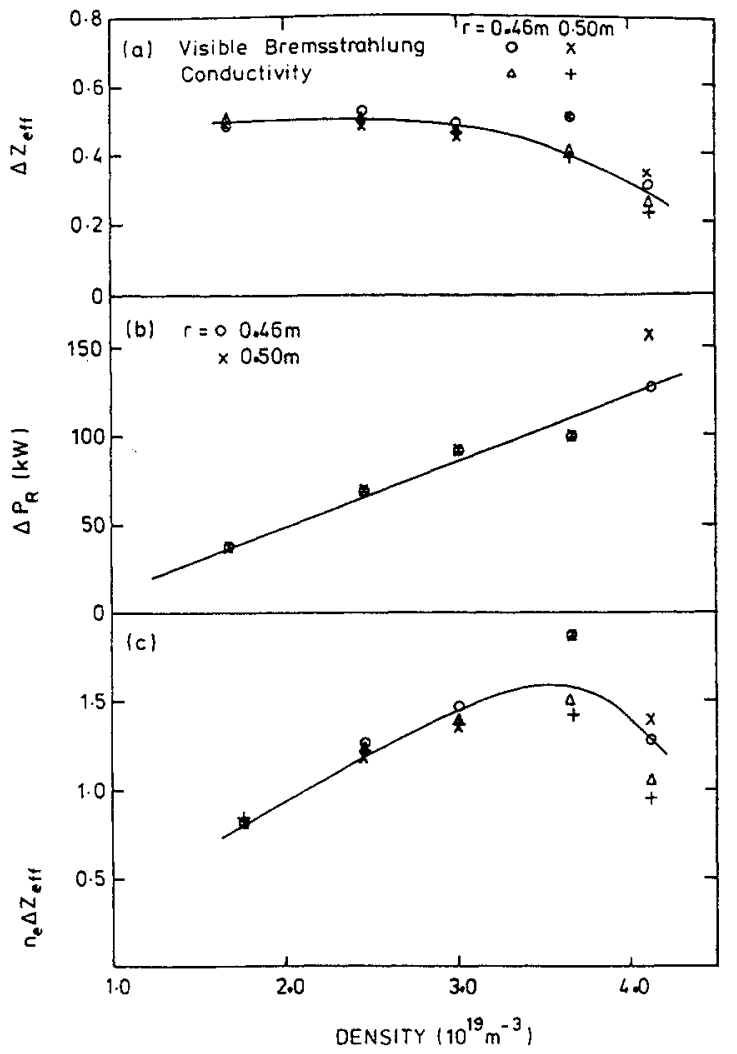

FIG. 7. (a) $\Delta \mathrm{Z}_{\text {eff }}$ versus density for different radii of gas injection, (b) $P_{R}$ versus density for different radii of gas injection and (c) impurity concentration $\mathrm{n}_{m}$ calculated from $\mathrm{n}_{e}$ and $\Delta \mathrm{Z}_{\text {eff }}$ for different radii of gas injection.

Results from a series of discharges at different densities and two different radial positions are summarized in Fig. 7. The change in $Z_{\text {eff }}$ due to impurity injection was typically 0.5 , falling slightly as the density increased. However, no change was detected in $\Delta Z_{\text {eff }}$ between the two different radii of $r=0.50 \mathrm{~m}$ and $r=0.46 \mathrm{~m}$.

Two measurements of $Z_{\text {eff }}$ have been made. The first uses the loop voltage $V_{\mathrm{L}}$ and the central temperature measured by the ECE diagnostic to measure the central $Z_{\text {eff }}$ assuming that the central safety factor $q(0)=0.8$, based on polarimetry experiments in similar discharges [22]. A second, independent, method uses measurements of visible bremsstrahlung [19], which gives a spatially averaged measurement of $\boldsymbol{Z}_{\text {eff }}$. In general, there is good agreement between the two techniques in ohmically heated discharges. An estimate of the number of impurities can be obtained if it is assumed that the $Z_{\text {eff }}$ is constant across the plasma radius. The impurity concentration calculated from the product $n_{\mathrm{e}} \Delta Z_{\text {eff }}$ is plotted in Fig. 7(c). This increases by almost a factor of 2 as the density increases from $1.6 \times 10^{19}$ to $3.6 \times 10^{19} \mathrm{~m}^{-3}$, but decreases again for the highest density, close to the detached plasma condition [23].

Measurements of the increase of the total radiation $\Delta P_{R}$ in the same shots are shown in Fig. 7(b). This is seen to increase linearly with density. The slope of the line gives the radiative rate for neon ions entering the plasma. If a simple global model is assumed we may write

$\Delta P_{R}=\int n_{\mathrm{e}}(r) n_{\mathrm{m}}(r) L\left(T, \tau_{\mathrm{p}}\right) \mathrm{d} V$

where $n_{\mathrm{m}}$ is the impurity concentration and $L\left(T, \tau_{\mathrm{p}}\right)$ is the radiation function for a given impurity. Taking average values of electron and impurity ion density we obtain

$\bar{L}(T)=\frac{\Delta P_{R}}{\bar{n}_{\mathrm{e}} n_{\mathrm{m}} V}$

From the data in Fig. 7 we obtain $\bar{L}(T) \simeq 3 \times 10^{-33} \mathrm{~W} \cdot \mathrm{m}^{3}$, which is a factor of 2 lower than the theoretical value obtained by Carolan and Walsh [24] for $T_{\mathrm{e}}=1 \mathrm{keV}$, $n \tau_{\mathrm{p}}=10^{18} \mathrm{~m}^{-3} \cdot \mathrm{s}$. This is satisfactory agreement, considering the errors in the estimation of the impurity content and in $\tau_{\mathrm{p}}$.

Spectroscopic measurements of the charge states $\mathrm{Ne}$ I and Ne VIII have been made at the two different radii for gas injection. The time evolution of these signals is shown in Fig. 8, compared with $P_{R}$ and $Z_{\text {eff }}$. The increase in intensity of the Ne I and Ne VIII is also independent of the position of the gas source. Examination of the radial distribution of the total radiation shows that the maximum neon radiation comes from a radius of $\sim 0.33 \mathrm{~m}$.

The absolute fuelling rates have not been measured in these discharges since the gas puffing rate through the limiter port is not constant through the pulse. Approximate values using the integrated gas injection give a fuelling rate that is in reasonable agreement with the results obtained from the accurately calibrated gas source.

\subsection{Comparison of helium, neon and argon}

A series of experiments have been performed in which helium, neon and argon where puffed for a constant time of $0.5 \mathrm{~s}$ at an increasing rate into successive identical discharges. The time dependences of the three impurities are compared in Fig. 9. In order to compare helium, neon and argon directly, we use the total radiated power and $Z_{\text {eff }}$ rather than spectroscopic lines. As expected, the radiation in the helium case is very small, but the rise for neon is greater than that for 


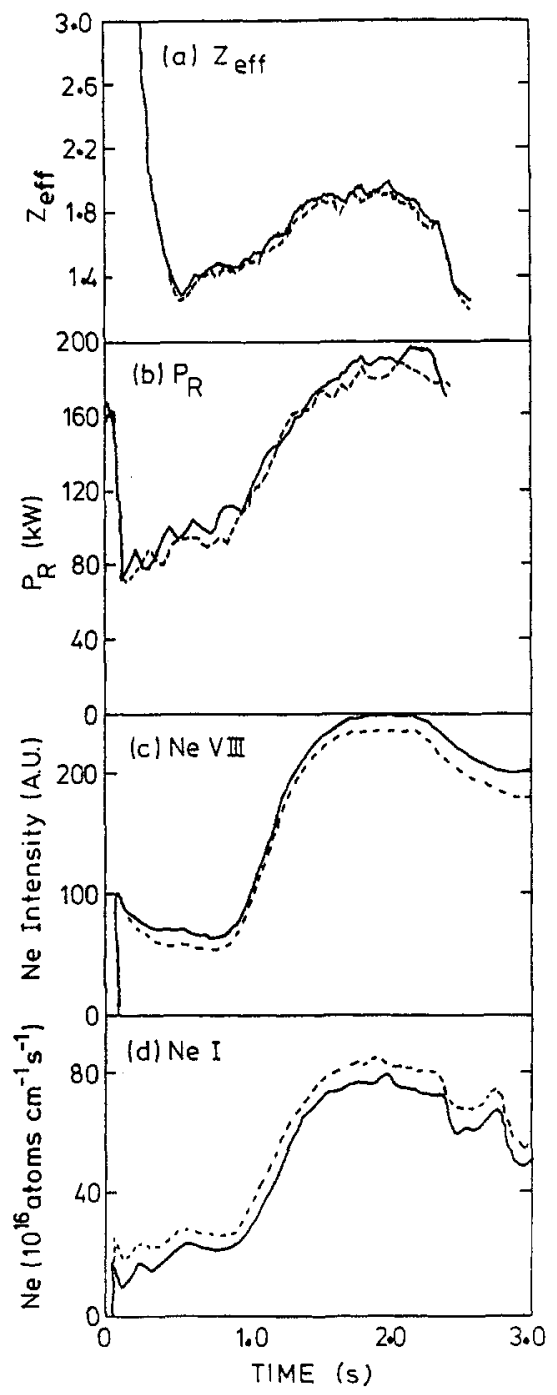

FIG. 8. Spectroscopic measurements of neon for gas sources at $0.46 \mathrm{~m}$ and $0.50 \mathrm{~m}$ in an Ohmic discharge at a density of $3 \times 10^{19} \mathrm{~m}^{-3}:$ (a) $\mathrm{Z}_{\text {effi }},\left(\right.$ b) $\mathrm{P}_{\mathrm{R}}$, (c) line integrated Ne VIII, (d) calibrated Ne I. In all parts, full curves for $0.46 \mathrm{~m}$ data and dashed curves for $0.50 \mathrm{~m}$ data.

argon despite the lower atomic number. The $Z_{\text {eff }}$ data, Fig. 9(b), show similarly that the rise for neon is greater than that for argon; although $\Delta Z_{\text {eff }}$ is lower, the helium makes a significant contribution to $Z_{\text {eff }}$. It should be noted that in other experiments where the initial $Z_{\text {eff }}$ is higher, for example those dominated by carbon or oxygen, that the introduction of helium is observed to make no change, or even to reduce $Z_{\text {eff }}$. Only when the plasma is initially very clean can the increase in $Z_{\text {eff }}$ be observed.

The results in Fig. 9 were obtained with the pumped limiter operating. In Fig. 10 results are compared with the ALT II pumps on and off. For helium and neon, the impurity concentration remains almost constant when the pumps are off indicating that $\tau_{p}^{*} \rightarrow \infty$, i.e. recycling is very close to $100 \%$. The $\tau_{\mathrm{p}}^{*}$ obtained when the pumps are on is thus a measure of the loss to the pumped limiter. In contrast, the value of $\tau_{\mathrm{p}}^{*}$ for argon is independent of whether the pump is on or off. This is discussed further in Section 5.3. The relative values of $\tau_{\mathrm{p}}^{*}$ (Table I) are quite similar to those obtained for helium, neon and argon by Ramsey and Manos in TFTR [25], even though the presence of the pumped limiter might be expected to alter the recycling conditions.
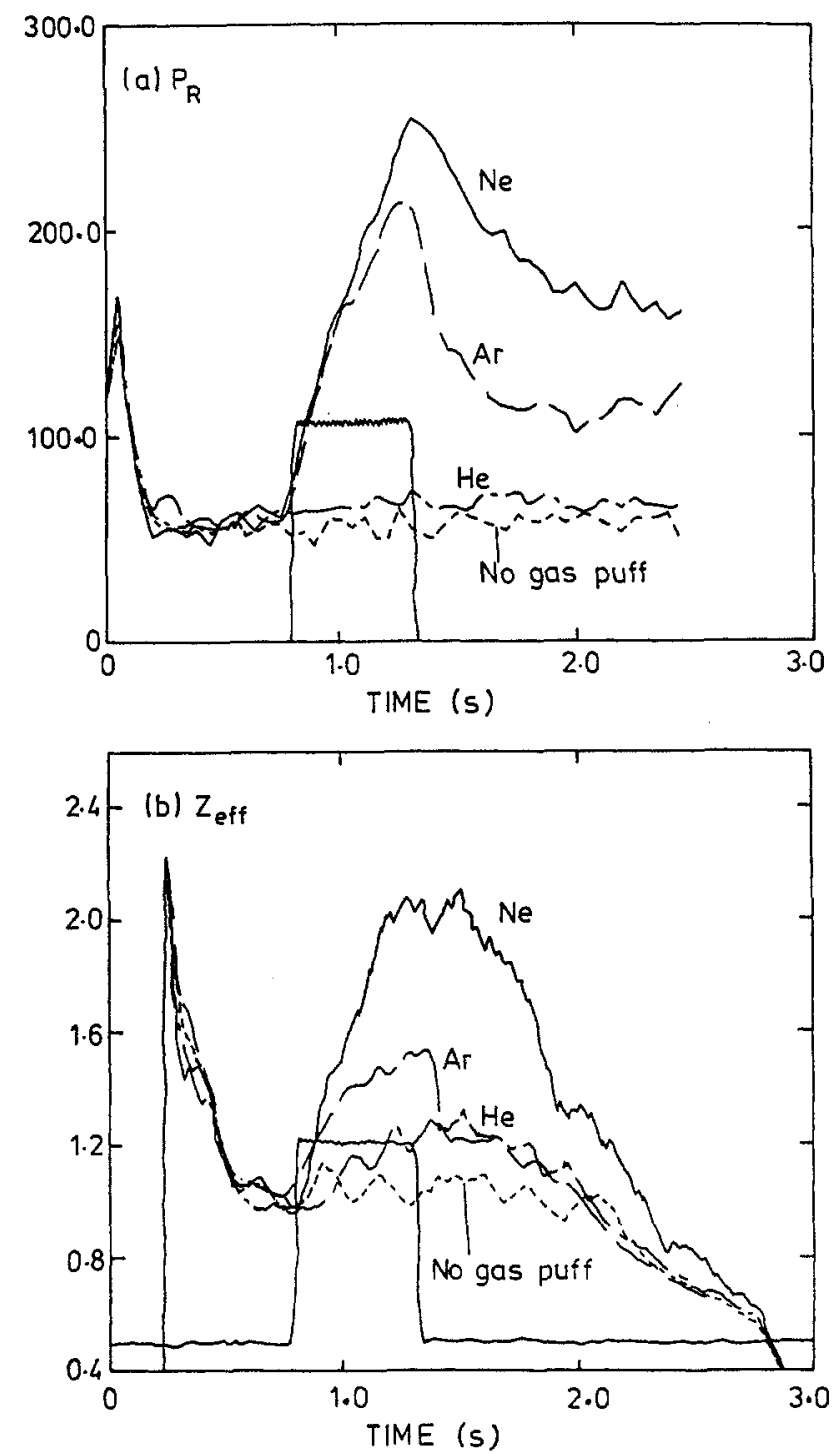

FIG. 9. Comparison of the time behaviour of (a) the radiation, $\mathrm{P}_{\mathrm{R}}$, and (b) $\mathrm{Z}_{\text {eff }}$ due to helium, neon and argon injected into three similar deuterium Ohmic discharges for $\mathrm{I}_{p}=340 \mathrm{kA}$ and $\mathrm{n}_{e}=2 \times 10^{19} \mathrm{~m}^{-3}$. Gas injection at $0.8 \mathrm{~s}$ for $0.5 \mathrm{~s}$ : helium $12.6 \times 10^{18}$ atoms, neon $3.7 \times 10^{18}$ atoms and argon 4.4. $\times 10^{18}$ atoms. 


\section{McCRACKEN et al.}
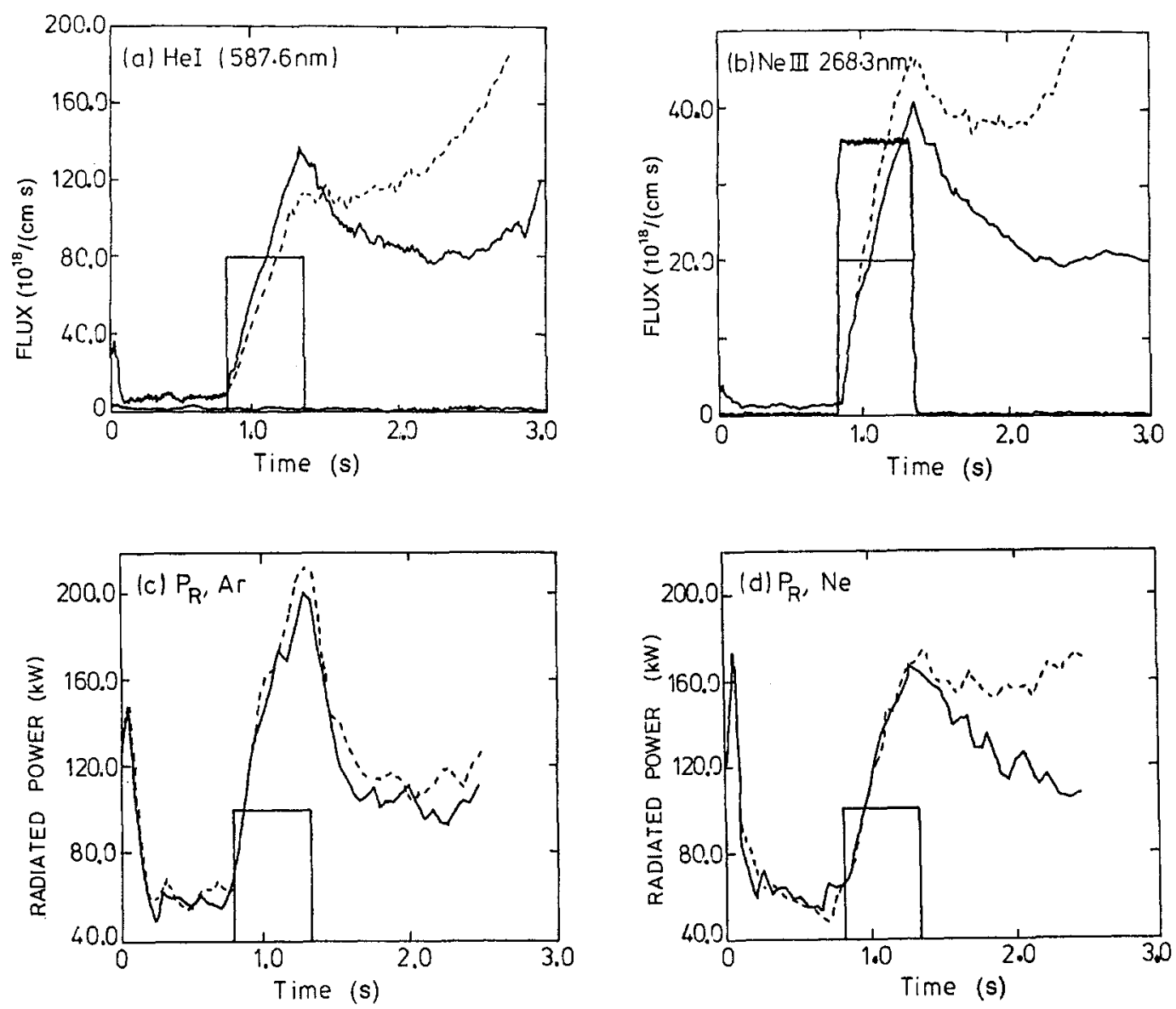

FIG. 10. Illustration of the different effective decay times $\tau_{p}^{*}$ with the ALT II pumped limiter on and off: (a) He I, (b) Ne III, (c) argon $\mathrm{P}_{\mathrm{R}}$, (d) neon $\mathrm{P}_{\mathrm{R}}$. In all parts full curves indicate pumps on and dashed curves indicate pumps off.

The increase in $P_{R}$ and $Z_{\text {eff }}$ with increasing gas flow rate is shown in Figs 11(a) and (b). It is seen that both $Z_{\text {eff }}$ and the radiated power increase linearly with the injected flux of atoms, $G$. From the rate of rise of $Z_{\text {eff }}$ and $P_{R}$ at $t=0$, we can in principle calculate the rate at which impurity atoms are actually entering the discharge, i.e. the source function $S$ in Eq. (1). The number of impurity atoms in the plasma has been estimated from $\Delta Z_{\text {eff }}$ assuming that $Z_{\text {eff }}$ is constant across the plasma radius. In Fig. 11(c) the number of atoms in the plasma at the end of gas injection is plotted as a function of the number of atoms injected. As indicated by the raw data for radiated power and $Z_{\text {eff }}$, the argon efficiency is much lower than that for neon. This low fuelling efficiency is even further depressed in its effect on the plasma through the smaller $\tau_{p}^{*}$, as observed in Fig. 10(c).

\subsection{Comparison of carbon monoxide and methane injection}

In practice, the most important impurities in plasmas are usually oxygen and carbon. Earlier experiments on the study of these impurities have concentrated on the local transport in the boundary layer both parallel and across the magnetic field [3-5]. In the present experiments we have used the same approach as with the rare gases to try to determine the effective fuelling rate. The observed time dependence of the spectroscopic signals during $\mathrm{CO}$ injection is shown in Fig. 12.

There is a striking difference between the behaviours of oxygen and carbon. Whereas the oxygen influx increases approximately linearly with time, indicating high recycling and effective integration of the impurity influx over the first $0.5 \mathrm{~s}$, the calibrated C I flux does 
TABLE I. SUMMARY OF EXPERIMENTAL RESULTS

\begin{tabular}{|c|c|c|c|c|c|c|c|c|c|}
\hline & $\begin{array}{c}n_{\mathrm{e}} \\
\left(19^{19} \mathrm{~m}^{-3}\right)\end{array}$ & $\begin{array}{l}\text { Influx in } \\
0.5 \mathrm{~s} \\
\left(10^{18}\right) \\
\pm 5 \%\end{array}$ & $\begin{array}{l}\text { Recycling flux } \\
\qquad \Gamma_{R} \\
\quad\left(10^{19} \mathrm{~s}^{-1}\right) \\
+100 \% \\
-50 \%\end{array}$ & $\begin{array}{c}\Delta Z_{\mathrm{eff}} \\
\pm 0.05\end{array}$ & $\begin{array}{c}\Delta P_{R} \\
(\mathrm{~kW}) \\
\pm 10\end{array}$ & $\begin{array}{c}S / G \\
(\%) \\
+20 \%\end{array}$ & $\begin{array}{c}\tau_{\mathrm{p}}^{*} \\
(\mathrm{~s}) \\
\pm 20 \%\end{array}$ & $\begin{array}{c}\quad R \\
(1-R) \\
+100 \% \\
-50 \%\end{array}$ & $\begin{array}{c}\tau_{\mathrm{p}} \\
(\mathrm{ms}) \\
+100 \% \\
-50 \%\end{array}$ \\
\hline $\mathrm{He}$ & 2 & 13 & 11 & 0.30 & $10 \pm 5$ & 85 & 1.65 & 0.940 & 100 \\
\hline He (ALT II off) & 2 & 13 & 10 & 0.33 & $10 \pm 5$ & 93 & $>10$ & 0.986 & $>137$ \\
\hline $\mathrm{Ne}$ & 2 & 2.0 & 5.2 & 0.53 & 105 & 38 & 1.15 & 0.990 & 12 \\
\hline $\mathrm{Ne}$ (ALT II off) & 2 & 2.0 & 5.5 & 0.60 & 110 & 45 & 3.4 & 0.996 & 12 \\
\hline Ar & 2 & 4.4 & - & 0.35 & 140 & 3.6 & $0.4-0.7$ & $(0.997)$ & $(1.5)$ \\
\hline Ar (ALT II off) & 2 & - & - & - & 150 & 4.3 & $0.4-0.7$ & $(0.997)$ & $(1.5)$ \\
\hline $\mathrm{O}(\mathrm{CO})$ & 2.85 & 20 & 1.9 & 0.74 & 98 & 7.0 & 0.41 & 0.846 & 65 \\
\hline $\mathrm{C}\left(\mathrm{CH}_{4}\right)$ & 3.4 & 20 & -0.33 & $<0.02$ & $5 \pm 5$ & - & - & $(0.35)$ & - \\
\hline \multicolumn{10}{|l|}{ Density scan } \\
\hline \multirow[t]{4}{*}{$\mathrm{Ne}$ (Ohmic) } & 1.6 & 1.06 & 1.1 & 0.7 & $45 \pm 5$ & 80 & 1.35 & 0.957 & 56 \\
\hline & 2.8 & 0.86 & 1.4 & 0.34 & 60 & 83 & 1.31 & 0.970 & 39 \\
\hline & 3.2 & 0.98 & 1.4 & 0.35 & 70 & 75 & 1.43 & 0.968 & 46 \\
\hline & 3.8 & 1.06 & 1.5 & 0.30 & 80 & 75 & 1.30 & 0.966 & 43 \\
\hline \multirow[t]{4}{*}{$\mathrm{Ne}(\mathrm{NBI})$} & 1.9 & 1.86 & 3.1 & 0.30 & 100 & 22 & 0.58 & 0.985 & 8.8 \\
\hline & 2.7 & 1.69 & 3.4 & 0.15 & 120 & 17 & 0.55 & 0.990 & 5.5 \\
\hline & 3.7 & 1.81 & 3.1 & 0.1 & 140 & 15 & 0.45 & 0.988 & 5.2 \\
\hline & 4.8 & 1.90 & 4.5 & 0.05 & 250 & 9 & 0.43 & 0.995 & 2.2 \\
\hline \multicolumn{10}{|l|}{ Steady state } \\
\hline $\mathrm{Ne}$ (Ohmic) & 3.9 & $0.65 \mathrm{~s}^{-1}$ & 1.8 & 0.65 & 160 & - & - & - & 100 \\
\hline $\mathrm{Ne}(\mathrm{NBI})$ & 2.9 & $3.4 \mathrm{~s}^{-1}$ & 7.2 & 0.25 & 240 & - & - & - & 7.4 \\
\hline $\mathrm{Ne}(\mathrm{NBI})$ & 4.3 & $2.2 \mathrm{~s}^{-1}$ & 3.9 & $<0.05$ & 210 & - & - & - & $<4$ \\
\hline
\end{tabular}

Note: An estimate of typical experimental errors is given at the top of each column. Note that the error for the recycling coefficient is in $1-R$. Values in brackets are derived using theoretical results from the LIM code.

not increase. Similar results are obtained at two separate viewing locations, on the ALT II toroidal limiter and on the upper poloidal limiter. Direct viewing of the gas inlet with a visible spectrometer shows a nearly square wave form for C III $(\lambda=435.0 \mathrm{~nm}$, not shown) similar to the gas injection signal, confirming that the methane is entering the plasma and dissociating into carbon atoms. The results for C III $(\lambda=229.6 \mathrm{~nm})$ and $\mathrm{C} \mathrm{V}(\lambda=227.1 \mathrm{~nm})$ observed $135^{\circ}$ toroidally away from the gas injection point are shown in Fig. 12(d). The C III increases but the C V hardly changes.

The results for methane injection are shown in Fig. 13. The gas influx in molecules $\cdot \mathrm{s}^{-1}$ is chosen to be the same for $C O$. There is again no increase in C I. Using the $\Delta Z_{\text {eff }}$ we can, as with the rare gases, calculate the average fuelling efficiency over the $0.5 \mathrm{~s}$ injection. During the methane injection it is found that $\leq 1 \%$ of the number of carbon atoms injected are in the plasma. A completely independent method of estimating the fuelling is to use the change in the number of deuterium molecules injected, calculated from the feedback loop that maintains constant electron density. It is found that one molecule of methane replaces $1 \pm 0.1$ molecules of $D_{2}$, so that the methane is only one fifth as effective as deuterium in fuelling electrons. Considering only hydrogenic atoms, the $\mathrm{CH}_{4}$ molecule is half as efficient as $\mathrm{D}_{2}$ in electron fuelling under these conditions. The deuterium fuelling efficiency itself can, however, vary widely depending on the condition of the torus. 


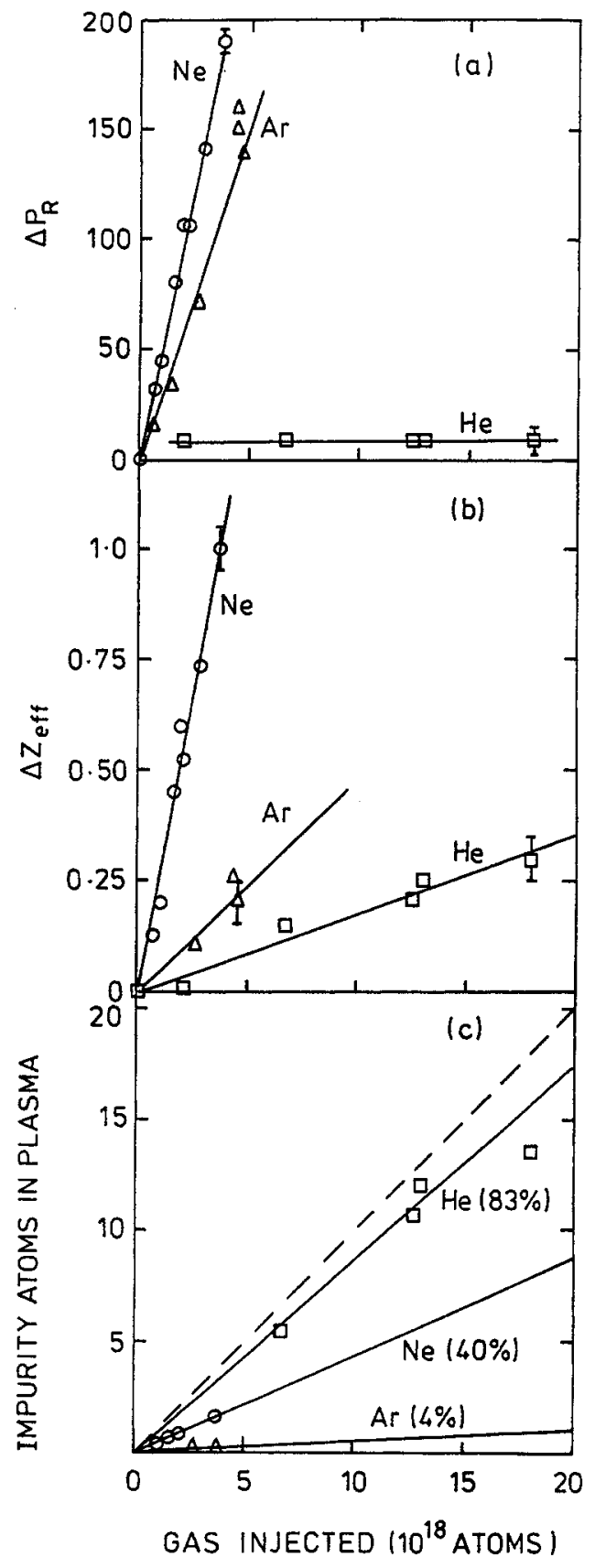

FIG. 11. (a) Power radiated and (b) $\mathrm{Z}_{\text {eff }} 0.5 s$ after the start of helium, neon and argon gas puffing into a deuterium discharge at various rates, and (c) $n_{e} \Delta Z_{\text {eff }}$ calculated from central value of $Z_{e f f}$ for helium, neon and argon injected into the same discharges.

If, on the basis of the evidence from the methane experiment and from Fig. 12, we assume that effectively no carbon enters the main confinement region during the CO puff, then from the $\Delta Z_{\text {eff }}$ value the fraction of the injected oxygen fluence that is in the plasma at the end of the $0.5 \mathrm{~s}$ injection period is $\sim 6 \%$.
The effect of radial position of the CO gas puff has been investigated using the puffing port in the test limiter. The results are shown in Fig. 14. Within the scatter of the results neither $P_{R}$ nor $Z_{\text {eff }}$ are strongly affected by the source position, just as had been observed for neon, Fig. 8. Calculations of the source function for ionization have been made for both $\mathrm{CO}$ and $\mathrm{CH}_{4}$. The case of $\mathrm{CO}$ is simpler because at the electron temperatures of interest the predominant reaction is ionization of the molecule [26]. This is followed by dissociation into oxygen and carbon atoms and ions [27]. The ionization source functions are similar to those of neon.

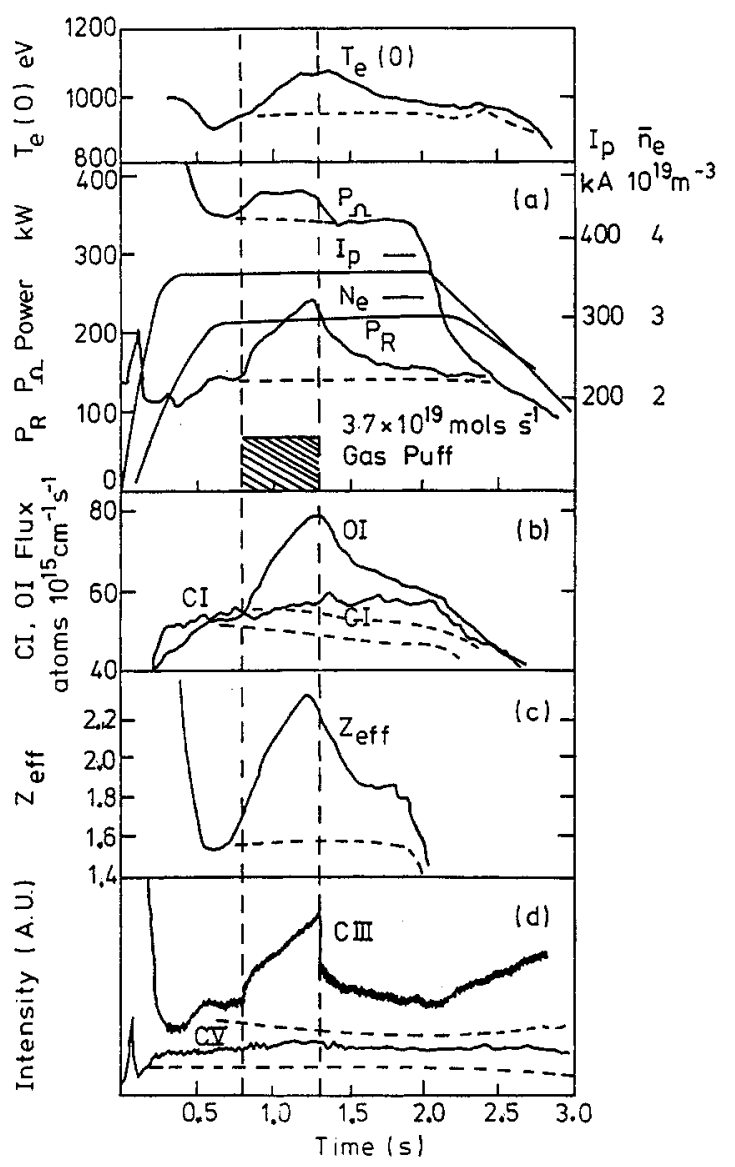

FIG. 12. Carbon monoxide gas injection into a $350 \mathrm{kA}$ ohmically heated plasma at a density of $2.8 \times 10^{19} \mathrm{~m}^{-3}$ using a flow rate of $3.7 \times 10^{19}$ molecules $\cdot s^{-1}$. Dashed curves indicate signals in the absence of a gas puff. 


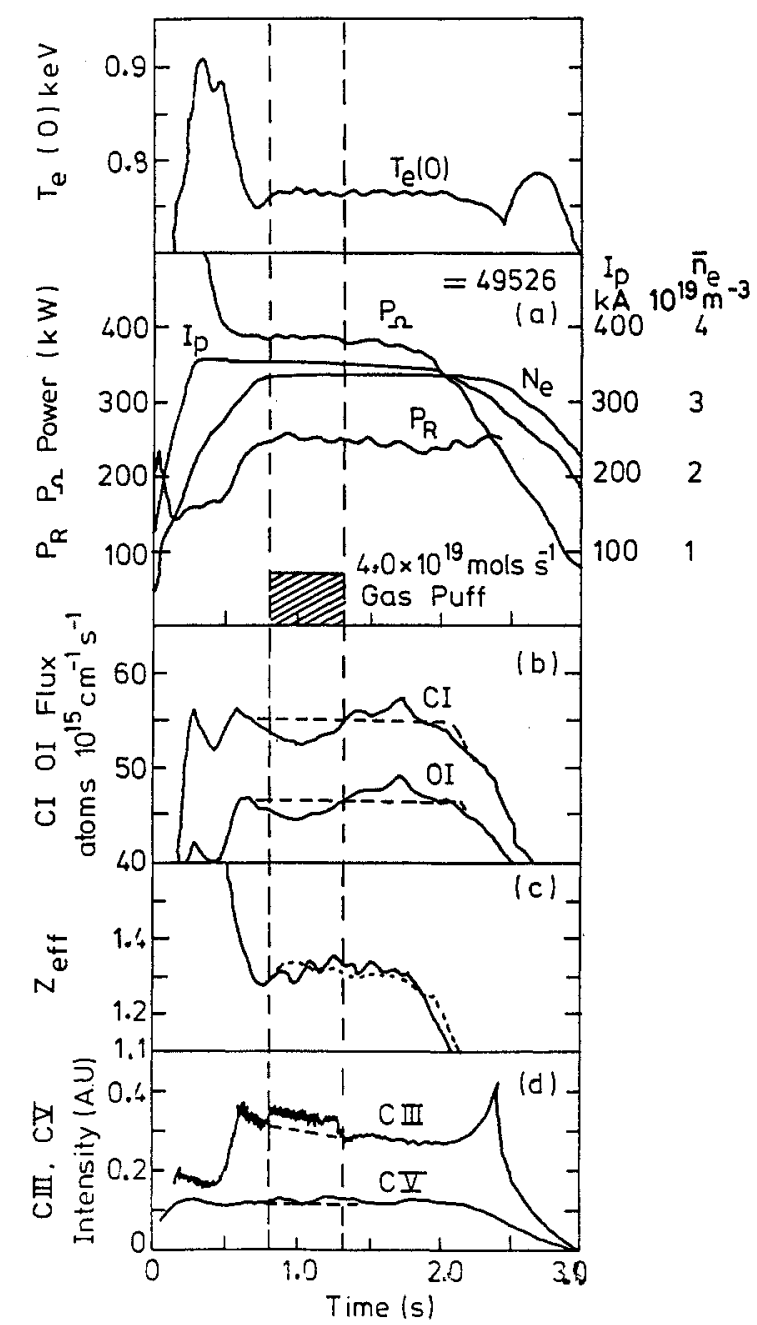

FIG. 13. Methane gas injection into a $350 \mathrm{kA}$ ohmically heated plasma at a density of $3.3 \times 10^{19} \mathrm{~m}^{-3}$ using a flow rate of $4.0 \times 10^{19}$ molecules $\cdot \mathrm{s}^{-1}$.

\section{DISCUSSION}

\subsection{Comparison of results for different impurity species}

The power radiated for all the different gas species as a function of the flow rate is plotted in Fig. 15. There is a tendency for the slope to increase, becoming nonlinear at higher flow rates. This is particularly noticeable in the data for argon and methane. Local measurements near the gas source show that the edge temperature is depressed by intense gas puffing [28]. This is expected to increase the photon efficiency, i.e. cause more radiation per ingoing atom. In our discussion of the present data we confine ourselves to conditions where the plasma is not disturbed by the gas puffing and $\Delta P_{R}$ is roughly proportional to the amount of gas injected.
A summary of the results for $\Delta Z_{\text {eff }}$ and $\Delta P_{R}$ for a given impurity influx is shown in Table I. The number of impurities in the discharge is calculated from $\Delta Z_{\text {eff }}$ by assuming that $Z_{\text {eff }}$ is constant with radius and taking a mean ion charge state based on the calculations of Carolan and Walsh [24] using the measured central temperature $T_{\mathrm{e}}(0)$. Only for argon is the mean charge significantly less than the atomic number.

The time constant $\tau_{\mathrm{p}}^{*}$ is calculated from the decay of the spectroscopic signals, $P_{R}$ and $Z_{\mathrm{eff}}$ at the end of the gas pulse, as shown in Fig. 3. The $\tau_{\mathrm{p}}^{*}$ from $P_{R}$ and the spectroscopic signals are, in general, in good agreement. For neon the decay is a good approximation to an exponential from 1.3 to $2.0 \mathrm{~s}$. Argon and helium tend to have a faster decay for the first $0.4 \mathrm{~s}$ and then become slower. The values of $\tau_{\mathrm{p}}^{*}$ from $Z_{\text {eff }}$ have large error bars because of high noise levels but seem to be consistently lower than the spectroscopic signals. This may be an indication that impurity transport in the plasma is significant. Since the radiation comes predominantly from large radii it will be expected to decay faster. The value of $Z_{\text {eff }}$ is determined more by the impurity content in the centre of the plasma where the impurities are most highly ionized. There is a significant difference in the $\tau_{\mathrm{p}}^{*}$ between the first results for neon (during the comparison with $\mathrm{He}$ and $\mathrm{Ar}$ ) and the results during the density scan. The two sets of measurements were taken at different times and thus the differences could be due to different wall conditionings [25].

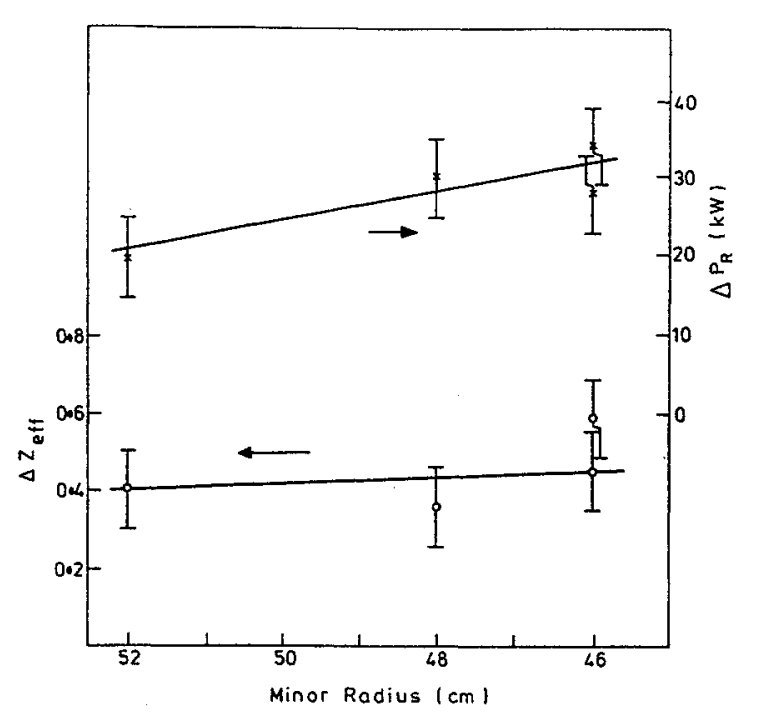

FIG. 14. Effect of varying the radial position of the gas puffing port on $\mathrm{Z}_{\text {eff }}$ and the radiated power in successive discharges using a constant gas puff between 0.5 and $1.3 \mathrm{~s}$. Measurements are made at $1.3 \mathrm{~s}$. 


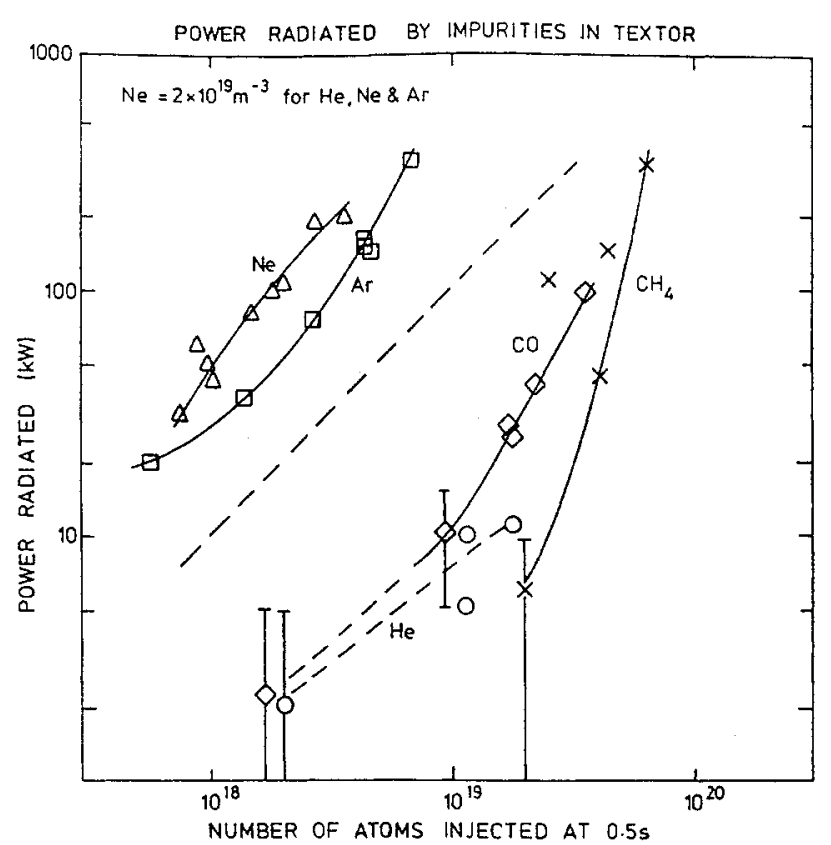

FIG. 15. Summary of the radiated power at the end of a $0.5 \mathrm{~s}$ gas puff as a function of puffing rate for $\mathrm{He}, \mathrm{Ne}, \mathrm{Ar}, \mathrm{CO}$ and $\mathrm{CH}_{4}$.

The effective source function $S$ is obtained in principle from the slope of the $\Delta Z_{\text {eff }}$ at $t=0$. In practice, because of the noise level on the $Z_{\text {eff }}$ signal, the value of $\Delta Z_{\text {eff }}$ at $t=0.5 \mathrm{~s}$ is taken and corrected for the finite $\tau_{\mathrm{p}}^{*}$, obtained from the spectroscopic signals. The correction factor is typically $\leq 1.3$ for the measured values of $\tau_{\mathrm{p}}^{*}$. The results of the ratio of source rate to gas influx rate, $S / G$, (see Section 2) are presented in Table I. The values of the effective source function divided by the primary influx, $S / G$, vary from $4 \%$ for argon to approximately $100 \%$ for both neon and helium. The value for oxygen is about twice that for argon. No value of $S / G$ can be calculated for carbon because there is no measurable increase for either $\Delta Z_{\text {eff }}$ or $\Delta P_{R}$, and hence a value of $\tau_{\mathrm{p}}^{*}$ cannot be obtained. The fuelling efficiency at $0.5 \mathrm{~s}$ is $<1 \%$. Whether this is due to a low effective source function $S$ or to a low $\tau_{\mathrm{p}}^{*}$ is not immediately obvious. This is discussed below. The value of the recycling coefficient $R$ is calculated in two ways.

The first method uses feedback control to obtain steady state conditions, as discussed in Section 4.1. The sum of the recycling flux $\Gamma_{R}$, and the primary influx $S$ is measured spectroscopically using a theoretical photon efficiency for a measured edge temperature. Then Eq. (1) is used. In the second method the ratio of the recycling flux to the primary flux $\Gamma_{R} / S$ is required and Eq. (5) is applied for any arbitrary time.
The value of $\Gamma_{R}$ is measured spectroscopically. The value of $S$ is obtained from the gas puffing rate and the average values of $S / G$. The steady state method is more accurate for large values of $R$, as the recycling flux dominates. However, it does not allow $\tau_{\mathrm{p}}^{*}$ or $R$ to be measured in the same discharge. The ratio method has an additional error in the measurement of $S$, particularly where $S / G \ll 1$. The total recycling influx is obtained by integrating the local calibrated signal for $\mathrm{He}$ I, Ne I and O I over the total length of the ALT II toroidal limiter. The presence of this limiter makes such an integration more reliable than in the case of poloidal limiters. There is, however, an uncertainty in the recycling flux of at least a factor of 2 due to the uncertainty in the theoretical photon efficiency used to obtain the particle flux from the spectroscopic line intensity. From the values of $R$ and $\tau_{\mathrm{p}}^{*}$ a value of $\tau_{\mathrm{p}}$ is obtained from Eq. (2).

The results are shown in Table I. The results for the recycling coefficient of helium are in reasonable agreement with those of earlier estimates [8]. The error in the absolute value of the recycling flux $\Gamma_{R}$ leads to a proportional error in $\tau_{\mathrm{p}}$ and in $1-R$. Note that it is $1-R$ that is measured experimentally and the errors are in $1-R$ rather than $R$. It is seen that the oxygen recycling coefficient is significantly less than those for the rare gases. The values of the recycling coefficients of neon and helium are significantly lower when the pumps are on than when they are off. This simply illustrates the pumping action of the ALT II limiter. The $\tau_{\mathrm{p}}^{*}$ for argon is identical whether the pumps are on or off, as stated earlier. For argon there is no absolute measure for the recycling flux and so we have used the theoretical $\tau_{\mathrm{p}}$ from LIM (Table II) together with experimental $\tau_{\mathrm{p}}^{*}$ to obtain $R$. It is observed to be similar to the values for neon or helium. This indicates that it is the short confinement time, $\tau_{\mathrm{p}}$, that causes the short $\tau_{\mathrm{p}}^{*}$ in argon, rather than a low value of $R$. The short $\tau_{\mathrm{p}}$, together with $R<1$, lead to pumping of argon whether the ALT II pumps are on or off.

The examples of neon injection at different densities show that within experimental error neither $R$ nor $\tau_{\mathrm{p}}$ vary with density. In comparing the beam heated data with the Ohmic data it appears that $\tau_{\mathrm{p}}$ decreases by a factor of about 4 . The results from the steady state method and the ratio method agree within a factor of about 2 .

\subsection{LIM code calculations}

Having independent measures of $\tau_{\mathrm{p}}$ and $\tau_{\mathrm{p}}^{*}$ is important in the interpretation of the fuelling behaviour. In 
TABLE II. LIM CODE CALCULATIONS

$\left(D_{\perp}=0.25 \mathrm{~m}^{2} \cdot \mathrm{s}^{-1}, V_{\mathrm{p}}=-2 D_{\perp} r / a^{2}\right)$

\begin{tabular}{|c|c|c|c|c|c|c|c|}
\hline & $\begin{array}{c}E_{0} \\
(\mathrm{eV})\end{array}$ & $n_{\mathrm{e}}$ & $z$ & $\begin{array}{c}\tau_{\mathrm{p}} \\
(\mathrm{ms})\end{array}$ & $\begin{array}{c}P_{R 1} \\
\left(10^{-16} \mathrm{~W}\right)\end{array}$ & $\begin{array}{c}\varphi \\
\left(10^{19} \mathrm{~s}^{-1}\right) \\
(\text { exp. })\end{array}$ & $\begin{array}{c}\Delta P_{R} \\
(\mathrm{~kW})\end{array}$ \\
\hline \multirow[t]{2}{*}{$\mathrm{He}$} & 0.1 & 2.0 & 2 & 34 & 0.53 & 11 & 5.8 \\
\hline & $0.05 / 50$ & 2.0 & 2 & 40 & 0.53 & 11 & 5.8 \\
\hline $\mathrm{Ne}$ & 0.1 & 2.0 & 10 & 15 & 24 & 5.2 & 130 \\
\hline $\mathrm{Ar}$ & 0.03 & 2.0 & 16 & 1.5 & 6.6 & - & - \\
\hline $\mathrm{O}$ & 0.05 & 2.85 & 8 & 5.4 & 5.8 & 1.9 & 11 \\
\hline $\mathrm{O}$ & 10 & 2.85 & 8 & 36 & 30 & 1.9 & 56 \\
\hline o & 100 & 2.85 & 8 & 64 & 45.4 & 1.9 & 86 \\
\hline C & 0.05 & 2.0 & 6 & 13 & 5.0 & - & - \\
\hline \multicolumn{8}{|c|}{ Ohmic density scans } \\
\hline $\mathrm{Ne}$ & 0.1 & 1.6 & 10 & 22 & 31 & 1.1 & 35 \\
\hline $\mathrm{Ne}$ & 0.1 & 2.8 & 10 & 26. & 46 & 1.4 & 66 \\
\hline $\mathrm{Ne}$ & 0.1 & 3.2 & 10 & 16 & 50 & 1.4 & 70 \\
\hline $\mathrm{Ne}$ & 0.1 & 3.8 & 10 & 23 & 81 & 1.5 & 124 \\
\hline \multicolumn{8}{|c|}{ NBI } \\
\hline $\mathrm{Ne}$ & 0.1 & 1.9 & 10 & 20 & 28 & 3.4 & 97 \\
\hline $\mathrm{Ne}$ & 0.1 & 2.7 & 10 & 7.2 & 13 & 3.9 & 50 \\
\hline $\mathrm{Ne}$ & 0.1 & 3.7 & 10 & 5.1 & 20 & 3.6 & 70 \\
\hline $\mathrm{Ne}$ & 0.1 & 4.8 & 10 & 3.8 & 17 & 14 & 76 \\
\hline
\end{tabular}

principle, $\tau_{p}$ can be readily calculated. To illustrate the basic physics we consider the 1-D Engelhardt model [5] where the flux of a species leaving the plasma, $\Gamma$, is given by

$\Gamma=D_{\perp} A_{\mathrm{p}} n_{\mathrm{m}} / \lambda_{\mathrm{i}}$

where $D_{\perp}$ is the cross-field diffusion coefficient, $A_{\mathrm{p}}$ is the plasma surface area and $\lambda_{i}$ is the ionization mean free path. In its simplest form, discussed here, the model assumes a delta function source for the ions at a distance $\lambda_{i}$ inboard of the LCFS and no inward pinch, $v_{p}=0$. From Eq. (8) it follows that, for cylindrical geometry, the global impurity confinement time $\tau_{\mathrm{p}}$ can be expressed as

$\tau_{\mathrm{p}}=\frac{a v_{0}}{2 D_{\perp}(\sigma v) n_{\mathrm{e}}}$

where $v_{0}$ is the velocity of the impurity neutral entering the plasma, $\langle\sigma v\rangle$ is the ionization rate coefficient and $a$ is the minor radius of the plasma.

More detailed calculations can be performed using the Monte Carlo code LIM [29]. This has been extensively used for interpretative studies of JET [30], TFTR
[31], DITE [32] and previously also for TEXTOR results [4]. Experimental profiles of density and temperature of the background plasma are used as inputs to the code, together with details of the limiter shape, plasma radius and SOL connection length. Impurity atoms are launched with a predetermined energy distribution and each neutral is followed in 2-D until it is ionized. The ion is then followed in its parallel (assumed classical) and cross-field (assumed anomalous) transport. Further ionization and/or recombination and thermalization with the background ions continues according to Spitzer rates until the test particle reaches the wall or returns to the limiter. For purposes of comparison with spectroscopic data the code calculates the distribution of line emissions from transitions of interest. An atomic physics package [33] has been used. By integrating over all transitions of interest the total power radiated per recycling particle is calculated. The mean residence time of the impurity in the plasma, $\tau_{\mathrm{p}}$, is also obtained.

Edge profiles of $n_{\mathrm{e}}$ and $T_{\mathrm{e}}$ have been measured for these discharges using the lithium, carbon and helium beams together with Langmuir probe data. These have been combined with the central profiles measured using the interferometer and ECE systems. The central and edge profiles typically match well at a radius $r=0.4 \mathrm{~m}$. A range of values of anomalous diffusion coefficient and pinch velocity $v_{\mathrm{p}}$ have been used in modelling the data. These, together with the velocity of the entering impurity species are the main fitting parameters, cf. Eqs (8) and (9). An incident energy of $0.1 \mathrm{eV}$ was chosen as the starting point for the neutral energy. In the case of helium, because of its low mass, there is an appreciable probability of backscattering $(\sim 8 \%)$ of the recycling ions. The backscattered ions have a mean energy of $50 \mathrm{eV}$. Inclusion of backscattering does not significantly affect the results.

Values of $D_{\perp}=0.25 \mathrm{~m}^{2} \cdot \mathrm{s}^{-1}$ and $v_{\mathrm{p}}=-2 D_{\perp} r / \mathrm{a}^{2}$ give a reasonable fit to the data. The chosen value of $D_{\perp}$ compares reasonably well with the value of $0.37 \mathrm{~m}^{2} \cdot \mathrm{s}^{-1}$ derived from the density profile in the SOL for Ohmic discharges. The results for these conditions are shown in Table II. Values of $D_{\perp}=1 \mathrm{~m}^{2} \cdot \mathrm{s}^{-1}$ and $v_{\mathrm{p}}=0$ have also been used, but with much poorer agreement with experiment. We have used the same value of $D_{\perp}$ for all impurity species based firstly on experimental evidence and secondly on the theoretical argument of Fussman et al. [10] that if anomalous diffusion is due to electrostatic fluctuations then it should be independent of mass. Experimental data obtained with injected impurities show that the flux e-folding distance in the SOL is independent of mass over the range from hydrogen to platinum $[34,35]$. 
Considering the errors in the experimental fluxes the values of $\tau_{\mathrm{p}}$ from Table I are in reasonable agreement with the theoretical values, Table II. The theoretical values are typically a factor of 2 to 3 lower than experiment for the Ohmic discharges with helium and neon. In the beam heated discharges the $\Delta Z_{\text {eff }}$ values are less reliable so that the calculated fuelling rate $S$ has larger errors. This value has to be used to obtain $R$ and hence $\tau_{\mathrm{p}}$. Nevertheless, the results are in reasonable agreement with the LIM code calculations. The theoretical values of $\tau_{\mathrm{p}}$ are lower for the beam heated discharges because of the high measured edge temperature and the consequent lower ionization mean free path. The change in $\tau_{\mathrm{p}}$ rather than in $R$ is therefore responsible for the difference in the experimentally measured value of $\tau_{\mathrm{p}}$.

The case of oxygen appears anomalous. For $E_{0}=0.1 \mathrm{eV}$ the value of $\tau_{\mathrm{p}}$ is much lower than observed in experiment. This is almost certainly due to the high charge exchange cross-section of oxygen with hydrogen [36, 37]. Ionized oxygen atoms become neutralized and are no longer affected by the magnetic field. This results in them 'walking' more deeply into the discharge. In a crude attempt to account for this we have increased the value of $E_{0}$ to 10 and $100 \mathrm{eV}$, Table II. A value of $100 \mathrm{eV}$ gives results in reasonable agreement with experiment.

From the LIM calculation we obtain the power radiated per average single transit of an impurity atom into the plasma, $P_{R 1}$, Table II. In order to obtain the radiated power we use the total influx from experiment, Table I, and the power lost per particle entering, $P_{R 1}$. The radiated powers for helium and neon in Ohmic discharges are within a factor of 2 of the experimental values, which is well inside the experimental and spectroscopic data errors. The calculated values for the neutral beam heated neon cases are a factor of 2 to 3 too low compared with the experimental values. The theoretical result for oxygen is again anomalous, when an energy of $0.05 \mathrm{eV}$ is used for the impurity atom, but is in quite good agreement for an energy of $10-100 \mathrm{eV}$.

\subsection{Impurity recycling}

The impurity recycling coefficients should not be considered to be the result of a simple backscattering process [38]. Some impurity ions may become trapped in the surface and then, subsequently, be released by the impact of further incident impurity ions or by the main plasma species itself. It is therefore possible for an inventory of impurity atoms to build up in the surface and to be released for some time after the primary source has stopped. This could lead to another long time constant in the system, as is observed for tokamak surfaces during hydrogen isotope changeover experiments [39]. The situation is further complicated by the ion flux varying as a function of radius from the limiter edge out to the wall. Thus, there could be a wide range of time constants involved in the buildup and depletion of the impurity in the surface. Fortunately, in the case of the rare gases it turns out experimentally that these time constants appear to be short, less than or comparable with those determined by the plasma processes.

In the case of oxygen the relatively long $\tau_{\mathrm{p}}$ and shorter $\tau_{\mathrm{p}}^{*}$ result in a calculated recycling coefficient significantly less than those of the rare gases at $\sim 0.85$. This value is much higher than the expected backscattering coefficient [38]. It is not clear, however, whether it is due to thermal desorption or to ion induced release. Compared with the gaseous species, carbon might be expected to be different, as is observed experimentally. The effective recycling coefficient would be expected to be the sum of the true backscattering coefficient which is $\sim 10^{-2}$ [38], plus the self-sputtering yield, which can vary from 0.1 to 0.5 at normal incidence in the energy range of interest $(0.1-5 \mathrm{keV})$ [40]. The charge distribution and energy of the impurity ions returning to the limiter are calculated from LIM and a value of 0.35 for the self-sputter yield is obtained. Using calculated values of $R$ and $\tau_{\mathrm{p}}$ we deduce $\tau_{\mathrm{p}}^{*}$ to be $19.5 \mathrm{~ms}$. Examining Eq. (3) we note that the low value of $\tau_{\mathrm{p}}^{*}$ leads to a proportionally low impurity level, consistent with experiment. Because they have higher mean energies ( $\geq 5 \mathrm{eV}$ ) physically sputtered atoms will have larger ionization mean free paths than thermal atoms and hence larger values of $\tau_{\mathrm{p}}$ (Eq. (9)). The value of $\tau_{\mathrm{p}}$ for oxygen would still be expected to be larger than that for carbon, though by a smaller factor than in the case of thermal species.

Laboratory experiments have shown that chemical sputtering of carbon by oxygen produces a $\mathrm{CO}$ molecule with a yield of $\sim 1.0$ [41]. Clear evidence of oxygen sputtering of carbon has also been observed in the DITE tokamak when oxygen was deliberately introduced through a probe limiter [42]. This seems to be in apparent disagreement with similar experiments in TEXTOR. Differences between the two experiments were that TEXTOR was boronized and had a low intrinsic oxygen level whereas in DITE it was high, $\sim 5 \%$. Secondly, the observations of carbon sputtering in DITE were made at the probe limiter where the gas was injected, whereas in TEXTOR the observations are made on the main toroidal limiter $70^{\circ}$ toroidally from the gas source. During combined 
hydrogen and oxygen ion bombardment it has been shown that oxygen can be released from the surface as water [43] and there is recent evidence that physical sputtering of oxygen may occur [44]. There is thus some supporting evidence for the experimental result of Fig. 12 that the influx of oxygen is much greater than that of carbon and hence the yield for production of $\mathrm{CO}$ by oxygen impact must be $\ll 1$.

These observations are a remarkable illustration of the difference in behaviour of high recycling and low recycling impurities. Similar examples of low recycling impurities recently reported are the injection of lithium pellets into TFTR [45] and the injection of trimethyl boron into TEXTOR [46]. The TEXTOR experiment showed fuelling of the plasma by hydrogen without contamination by either boron or carbon.

The model discussed and the experimental results presented here illustrate the difficulty of defining a 'fuelling efficiency'. The fraction of the injected impurities present in the plasma is a function of time and depends on $R$ and $\tau_{\mathrm{p}}$ (and thus on $D_{\perp}$ and the ionization mean free path [30]).

\subsection{Ionization source profiles}

We have obtained some experimental values for the recycling coefficient $R$ that are close to unity. In the particular case of argon this result seems at first sight inconsistent with the low effective fuelling rate $S / G$, see Table I. To investigate the reason for the variation in fuelling rate we have calculated the source function for the three cases of helium, neon and argon under the same plasma edge conditions. The ionization source function at a given radius $S(x)$ (using an origin starting at the wall) can be expressed as

$S(x)=\frac{\mathrm{d}}{\mathrm{d} x}\left[G \exp \left(-\int \frac{\overline{\sigma v}(x) n_{\mathrm{e}}(x)}{v_{0}} \mathrm{~d} x\right)\right]$

Using the recommended ionization rates $\overline{\sigma v}\left(T_{\mathrm{e}}\right)$ as a function of temperature $[47,48]$ and the experimental profiles, we have calculated $S(x)$ as a function of plasma radius for the three rare gases. The results for an ohmically heated discharge are shown in Fig. 16. It is clear that argon is ionized further out radially than neon. This is not only because the ionization rate is higher, but also because, for a given gas temperature, the argon velocity is lower since the mass is larger. The helium ions are ionized further in than the argon and neon ions, most of them radially inside the LCFS.

The ionization of the argon far outside the LCFS is not a complete explanation of the low effective fuelling

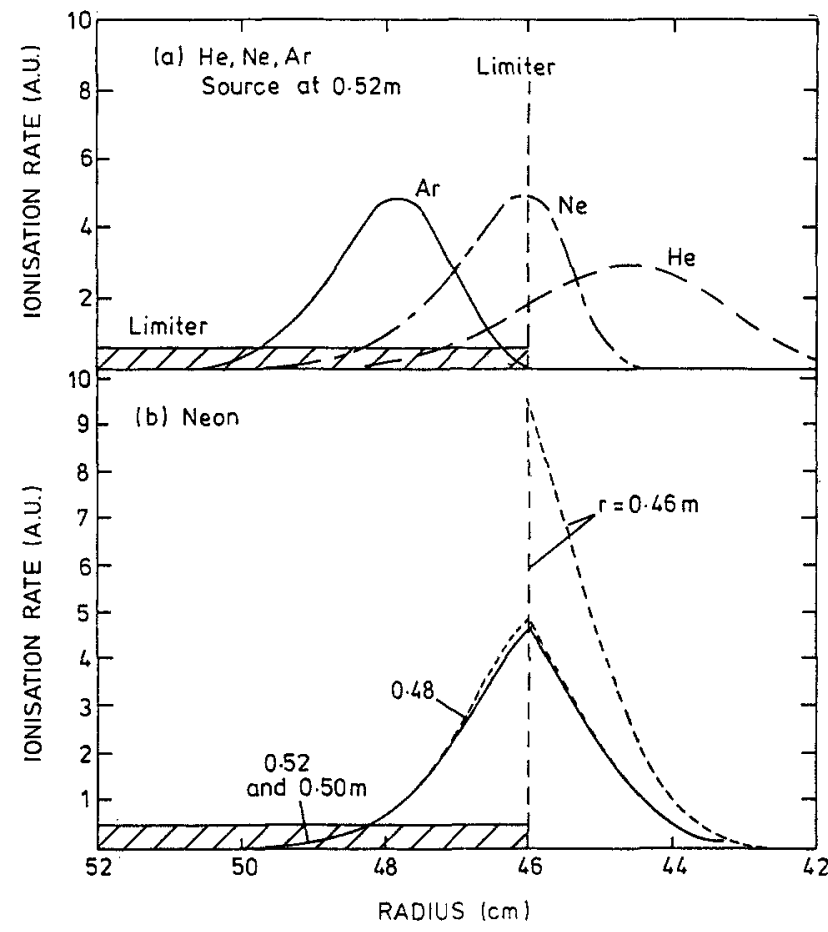

FIG. 16. Ionization source function calculated from a simple 1-D model (Eq. (8)) using experimental $\mathrm{n}_{e}$ and $\mathrm{T}_{e}$ profiles with atom energy $\mathrm{E}_{0}=0.05 \mathrm{eV}:(a)$ helium, neon and argon injected at $0.52 \mathrm{~m}$ with $\mathrm{n}_{e}=2.0 \times 10^{19} \mathrm{~m}^{-3}$; (b) neon injected at different radii with $\overline{\mathrm{n}}_{e}=2.8 \times 10^{19} \mathrm{~m}^{-3}$.

rate since argon atoms ionized in the SOL will in general hit the surface of the limiter or wall and recycle. By a series of such collisions they could eventually 'walk' in to the LCFS. There must, therefore, be some trapping in the surfaces of the vessel before the argon reaches the LCFS. Whether the low fuelling rate for argon is due to a larger number of collisions with the wall (due in turn to its high ionization cross-section) or to a higher trapping probability per wall collision cannot be deduced from the present data. We note again that the recycling coefficient for the outer surfaces could be much lower than that for the limiter edge near the plasma, which is subject to much higher ion fluxes and ion energies.

Similar calculations have been carried out for neon, carbon and oxygen for different primary source radii, to elucidate the results presented in Sections 4.2 and 4.4. The results for neon are shown in Fig. 16(b). It is seen that because of the steep gradients of plasma density and temperature there is essentially no difference between having the primary source positions between 0.52 and $0.48 \mathrm{~m}$. This is entirely consistent with the results in Figs 7 and 8 . At $0.46 \mathrm{~m}$ the calculated profile obviously changes. The fact that experimentally there is little change in the effective fuelling rate is 
probably because recycling dominates over the primary source. The ionization source functions for carbon and oxygen are similar to that for neon, with the peak in $S(x)$ about $10 \mathrm{~mm}$ further out.

The ionization source function has been calculated for neon for the different densities used in the experiments described in Section 4.1, Fig. 3. The decrease in edge temperature as the density increases results in the ionization source function remaining almost unchanged. This is consistent with both the experimental $\tau_{\mathrm{p}}^{*}$, Fig. 4 , and the LIM calculated $\tau_{\mathrm{p}}$ values, Table II, being approximately constant.

\section{CONCLUSIONS}

A range of gaseous impurity species have been injected into TEXTOR discharges and their effect on global plasma properties studied. A striking variation in their behaviour has been observed. The helium, neon and argon behave approximately as expected. These impurities are observed to have high recycling coefficients at the limiter so that the plasma acts as an integrator and over injection periods of up to $-1 \mathrm{~s}$ most of the impurities which reached the LCFS stayed in the plasma. There were, however, marked differences in the fuelling efficiencies. This is a consequence partly of the increasing ionization cross-section and lower injection velocity as the impurity mass increases. Each of these properties has the consequence that atoms entering from the wall have an increasing probability of being ionized on the first 'pass' through the SOL. If there was $100 \%$ reflection at all surfaces then the ions would have many chances to return to the plasma. The fact that the effective source function, $S$, is a small fraction of the incoming gas flow, particularly for argon, clearly indicates that there is some trapping of the species in the surfaces. The low effective source function for argon explains why, for a given primary source, the radiation is significantly lower than that for neon, which has a lower atomic number.

Carbon, injected both as carbon monoxide and methane, illustrates the case of the low recycling impurity. At the energy expected for ions returning to the limiter, true reflection is low $(\sim 1 \%)$ and so the effective reflection coefficient is determined by self-sputtering. Under typical conditions this is $\sim 0.3$. The low recycling results in a very much reduced impurity content. As the $\Delta Z_{\text {eff }}$ values observed were below detectable levels no direct measure of the effective source function could be made. If we assume it to be similar to that for oxygen then the impurity concentration and the radia- tion level are consistent with the effective recycling coefficient.

Finally, we consider oxygen. This appears to be a recycling impurity, showing a concentration initially increasing linearly with time. The effective source function ratio $S / G$ is $\sim 7 \%$, between the values for neon and argon, as expected on the basis of the ionization cross-sections. It is probable that oxygen is different to the rare gases in having a high sticking probability at the walls and other regions of the limiter - particularly when it arrives as an atom or atomic ion. Experimentally, oxygen is anomalous in the sense that $\tau_{\mathrm{p}}$ is much longer than the theoretical value for a thermal atom. This is attributed to the well known high charge exchange cross-section between $\mathrm{O}^{+}$and neutral hydrogen atoms. The high probability of charge exchange near the plasma edge is expected to increase the effective penetration energy for oxygen neutrals. This mechanism has been proposed to explain the large global confinement time which has been measured for oxygen.

Monte Carlo LIM code calculations have been carried out using the experimentally measured profiles of density and temperature. Detailed edge profiles are available from lithium, carbon and helium atom beams, in addition to Langmuir probes. Values of anomalous diffusion coefficient have been chosen as a fitting parameter and satisfactory agreement has been obtained for both particle replacement times and the radiated power with $D_{\perp}=0.25 \mathrm{~m}^{2} \cdot \mathrm{s}^{-1}$ and $v_{\mathrm{p}}=-2 D_{\perp} r / a^{2}$. This value of $D_{\perp}$ is consistent with the values of $0.35 \pm 0.20 \mathrm{~m}^{2} \cdot \mathrm{s}^{-1}$ estimated from the density profiles in the SOL and the value of $v_{\mathrm{p}}$ is consistent with the $n_{\mathrm{e}}$ profile. The same values of $D_{\perp}$ and inward pinch velocity have been chosen for all five impurity species studied, on the basis that anomalous diffusion is expected to be independent of mass.

The effect of varying plasma conditions on the fuelling rate has been studied in detail only for neon. Changing density has little effect on the number of impurity atoms in the discharge, although there is the expected linear increase in radiation and reduction in $Z_{\text {eff. }}$. One simple reason for this is that a lower edge temperature compensates for a higher density so that the mean free path for ionization changes little. The effect of varying the impurity source position also has surprisingly little effect. This has been studied in detail for both neon and $\mathrm{CO}$. Once the source is outside the radius at which significant ionization occurs, it is clear that its position is unimportant. Inside this radius the recycling appears to dominate and the primary source position is again not important. 
The present interpretation of fuelling efficiency has been performed with little consideration of transport in the core plasma. This is justified for high recycling impurities because the effective time constant $\tau_{\mathrm{p}}^{*}$ is long compared with the core long diffusion times in TEXTOR. In large devices it is expected that there will be a diffusion time for reaching radial equilibrium in the plasma itself so that it will be necessary to consider impurity transport in the core plasma in detail. However, it is clear from the experimental results and the LIM code analysis that the particle replacement time $\tau_{\mathrm{p}}$, which depends on $D_{\perp}, v_{p}$ and the ionization mean free path, together with the recycling coefficient play a major role in the fuelling efficiency.

\section{ACKNOWLEDGEMENTS}

We are grateful to Professor E. Hintz for his advice and encouragement of this work.

\section{REFERENCES}

[1] COHEN, S.A., et al., Phys. Rev. Lett. 35 (1975) 1507.

[2] PASINI, D., et al., Nucl. Fusion 30 (1990) 2049.

[3] PITCHER, C.S., et al., J. Nucl. Mater. 145-147 (1987) 596.

[4] McCRACKEN, G.M., et al., J. Nucl. Mater. 176\&177 (1990) 191

[5] STANGEBY, P.C., McCRACKEN, G.M., Nucl. Fusion 30 (1990) 1225.

[6] WEYNANTS, R.R., et al., Nucl. Fusion 32 (1992) 837.

[7] McCRACKEN, G.M., et al., Nucl. Fusion 18 (1978) 35.

[8] HILLIS, D.L., et al., Phys. Rev. Lett. 65 (1990) 2382.

[9] SYNAKOWSKI, E.J., et al., Phys. Rev. Lett. 65 (1990) 2255.

[10] FUSSMAN, G., et al., Plasma Phys. Control. Fusion 33 (1991) 1677.

[11] ENGELHARDT, W., et al., J. Nucl. Mater. 111\&112 (1982) 337.

[12] BERTSCHINGER, G., et al., Deutsche Physikalische Gesellschaft, Stuttgart, personal communication, 1988.

[13] SOLTWISCH, H., Plasma Phys. Control. Fusion 26 (1984) 23.

[14] DIPPEL, K.-H., et al., in Plasma Physics and Controlled Nuclear Fusion Research 1988 (Proc. 12th Int. Conf. Nice, 1988), Vol. 1, IAEA, Vienna (1989) 453.

[15] SAMM, U., et al., in Controlled Fusion and Plasma Physics (Proc. 18th Eur. Conf. Berlin, 1991), Vol. 15C, Part III European Physical Society, Geneva (1991) 157.

[16] POSPIESZCZYK, A., et al., Rev. Sci. Instrum. 59 (1988) 605.

[17] SCHWEER, B., et al., J. Nucl. Mater. 196-198 (1992) 174

[18] GOEBEL, D.M., et al., in Controlled Fusion and Plasma Heating (Proc. 15th Eur. Conf. Dubrovnik, 1988), Vol. 12B, Part II, European Physical Society, Geneva (1988) 667.
[19] ONGENA, J., et al., in Fusion Technology 1990 (Proc. 16th Symp. London, 1990), Vol. 1, Pergamon Press, Oxford (1991) 552.

[20] WINTER, J., et al., J. Nucl. Mater. 162-164 (1989) 713.

[21] SOBELMAN, I., et al., Excitation of Atoms and Broadening of Spectral Lines, Springer-Verlag, Berlin (1981).

[22] SOLTWISCH, H., Plasma Phys. Control. Fusion 34 (1992) 1669.

[23] SAMM, U., et al., Plasma Phys. Control. Fusion 29 (1987) 1321.

[24] CAROLAN, P.G., WALSH, M.J, AEA Fusion, Culham Laboratory, Abingdon, personal communication, 1991.

[25] RAMSEY, A.T., MANOS, D.M., J. Nucl. Mater. 196-198 (1992) 509.

[26] ORIENT, O.J., SRIVASTAVA, S.K., J. Phys., B At. Mol. Phys. 20 (1987) 3923.

[27] MITCHELL, J.B.A., HUS, H., J. Phys., B At. Mol. Phys. 18 (1985) 547.

[28] McCRACKEN, G.M., et al., J. Nucl. Mater. 196-198 (1992) 199.

[29] STANGEBY, P.C., et al., Nucl. Fusion 28 (1988) 1945.

[30] STANGEBY, P.C., J. Nucl. Mater. 176\&177 (1990) 51.

[31] PITCHER, C.S., et al., J. Nucl. Mater. 196-198 (1992) 241.

[32] PITCHER, C.S., et al., J. Nucl. Mater. 162-164 (1989) 337.

[33] VAN MAANEN-ABELS, A.E.P.M., A Package for Non Coronal Impurity Data, Int. Rep. JET-DN-T(85)29, JET, 1985.

[34] BAKOS, J.S., et al., J. Nucl. Mater. 162-164 (1989) 376.

[35] BAKOS, J.S., et al., J. Nucl. Mater. 162-164 (1989) 381.

[36] COHEN, S.A., DYLLA, H.F., Nucl. Fusion 18 (1978) 5.

[37] TENDLER, M., Plasma Phys. Control. Fusion 25 (1983) 767

[38] ECKSTEIN, W., in Atomic and Plasma-Material Interaction Data for Fusion (Suppl. to the Journal Nucl. Fusion), Vol. 1, IAEA, Vienna (1991) 17

[39] McCRACKEN, G.M., STOTT, P.E., Nucl. Fusion 19 (1979) 889.

[40] ROTH, J., J. Nucl. Mater. 176\&177 (1990) 132.

[41] ROTH, J., et al., in Atomic and Plasma-Material Interaction Data for Fusion (Suppl. to the Journal Nucl. Fusion), Vol. 1, IAEA, Vienna (1991) 63

[42] PITCHER, C.S., et al., Nucl. Fusion 29 (1989) 1919.

[43] ROTH, J., Max-Planck-Institut für Plasmaphysik, Garching, personal communication, 1992; VIETZKE, E., Forschungszentrum Jülich $\mathrm{GmbH}$, Jülich, personal communication, 1992.

[44] BOGEN, P., RUSBÜLDT, D., J. Nucl. Mater. 196-198 (1992) 179.

[45] SNIPES, J.A., et al., in Controlled Fusion and Plasma Physics (Proc. 18th Eur. Conf. Berlin, 1991), Vol. 15C, Part III, European Physical Society, Geneva (1991) 141.

[46] ESSER, H.G., et al., J. Nucl. Mater. 196-198 (1992) 231.

[47] BELL, K.L., et al., Recommended Cross Sections and Rates for Electron Ionisation of Light Atoms and Ions, Part I, Rep. CLM R216, Culham Laboratory, Abingdon, 1982.

[48] LENNON, M.A., et al., Recommended Cross Sections and Rates for Electron Ionisation of Atoms and Ions, Part II, Rep. CLM R270, Culham Laboratory, Abingdon, 1986.

(Manuscript received 15 February 1993

Final manuscript received 5 July 1993) 